\title{
Algebraic Signatures Enriched by Dependency Structure
}

\author{
Grzegorz Marczyński \\ gmarc@mimuw.edu.pl \\ Institute of Informatics, University of Warsaw
}

\begin{abstract}
Classical single-sorted algebraic signatures are defined as sets of operation symbols together with arities. In their many-sorted variant they also list sort symbols and use sort-sequences as operation types. An operation type not only indicates sorts of parameters, but also constitutes dependency between an operation and a set of sorts. In the paper we define algebraic signatures with dependency structures described as well-founded strict orders. We argue that the most natural morphisms between such structures are p-morphisms (short for pseudo-epimorphisms) and we prove that all together they constitute a category. In modeltheory structures like $\langle W, R\rangle$, where $W$ is a set and $R \subseteq W \times W$ is a transitive relation, are called transitive Kripke frames [Seg70]. Part of our result is a definition of a construction of non-empty products in the category of transitive Kripke frames. We prove that in general not all such products exist, but when the class of relations is limited to wellfounded strict orders, the category has all products of non-empty families of objects. We also show the existence of equalizers and its cocompletness.
\end{abstract}

\section{Introduction}

Classical single-sorted algebraic signatures are defined as sets of operation symbols together with arities. In their many-sorted variant they also list sort symbols and use sort-sequences as operation types. One should notice that an operation type not only indicates sorts of parameters, but also constitutes dependency between an operation and a set of sorts. Informally, one can reason that an operation cannot be defined unless all sort carriers from its type are present in the model.

In architectural approach to system specification [ST97], a signature represents a software module interface. The whole system (or, to be precise, its model) is obtained as a series of applications of so-called generic modules [BST99] also known as constructor implementations [ST88]. Modules are put together and constitute a whole only if all required parameter-modules are instantiated. Clearly this reveals a dependency relation between modules and, as a consequence, between operation symbols they define.

Our work on architectural models led us to a need of dependency structures put directly on operation symbols right in signatures. The idea is to extend the 
classical many-sorted signatures by explicitly defining the dependency of sorts and operations. Models over such signatures are the same as in the standard framework.

In the paper we define algebraic signatures with dependency structures described as well-founded strict orders. We argue that the most natural morphisms between such structures are p-morphisms (short for pseudo-epimorphisms) and we prove that all together they constitute a category. Unfortunately the category lacks the final object. However, we successfully show the existence of all products of non-empty families of objects and the presence of all equalizers. We also prove its cocompletness.

In model-theory structures like $\langle W, R\rangle$, where $W$ is a set and $R \subseteq W \times W$ is a relation, are called Kripke frames. It is standard to define categories of Kripke frames and p-morphisms [Seg70]. Part of our result is a definition of a construction of non-empty products in the category of transitive Kripke frames. We prove that in general not all such products exist, but when the class of relations is limited to well-founded strict orders, the category has all products of non-empty families of objects. We also show the existence of equalizers and its cocompletness.

Results presented in the paper are part of the ongoing work on the use of signature fragments to specify architecture of generic software modules.

The paper is organized as follows. First in Sect. 2 we present the motivation to our work. In Sect. 3 we define the categories of dependency relations. In Sections 4 and 5 we analyze the existence of colimits in these categories and in Sect. 6 we define the category of signatures with dependency structure and prove its properties. Finally, Sect. 7 contains conclusion and future work. Proofs of most lemmas and theorems are in the Appendix A of the extended version of this paper [Mar10].

\section{Motivation}

Classical algebraic many-sorted signatures naturally contain dependence of operation symbols on their parameters' sorts. In the architectural specifications [BST99], signature of a generic module is an injective signature morphism $\sigma: \Sigma_{\text {Form }} \rightarrow$ $\Sigma_{\text {Res }}$, where $\Sigma_{\text {Form }}$ is a formal parameters signature and $\Sigma_{\text {Res }}$ is a result signature. It renders a dependency between all symbols from the result signature and those from the parameters signature.

The above-described dependency is of the weak form, meaning that it is not required that actual implementation of result symbols uses the parameter symbols intrinsically. It rather conveys the negative information, leaving some symbols definitely independent of others. One may think of it as a of potential dependency.

The generic module application along a fitting morphism $\varphi: \Sigma_{F o r m} \rightarrow \Sigma_{A c t}$ on the signature-level is simply the pushout of $\varphi$ and $\sigma$. We notice that in the pushout signature the dependency of the result symbols on the parameter symbols is lost. Consider the following simple example. 


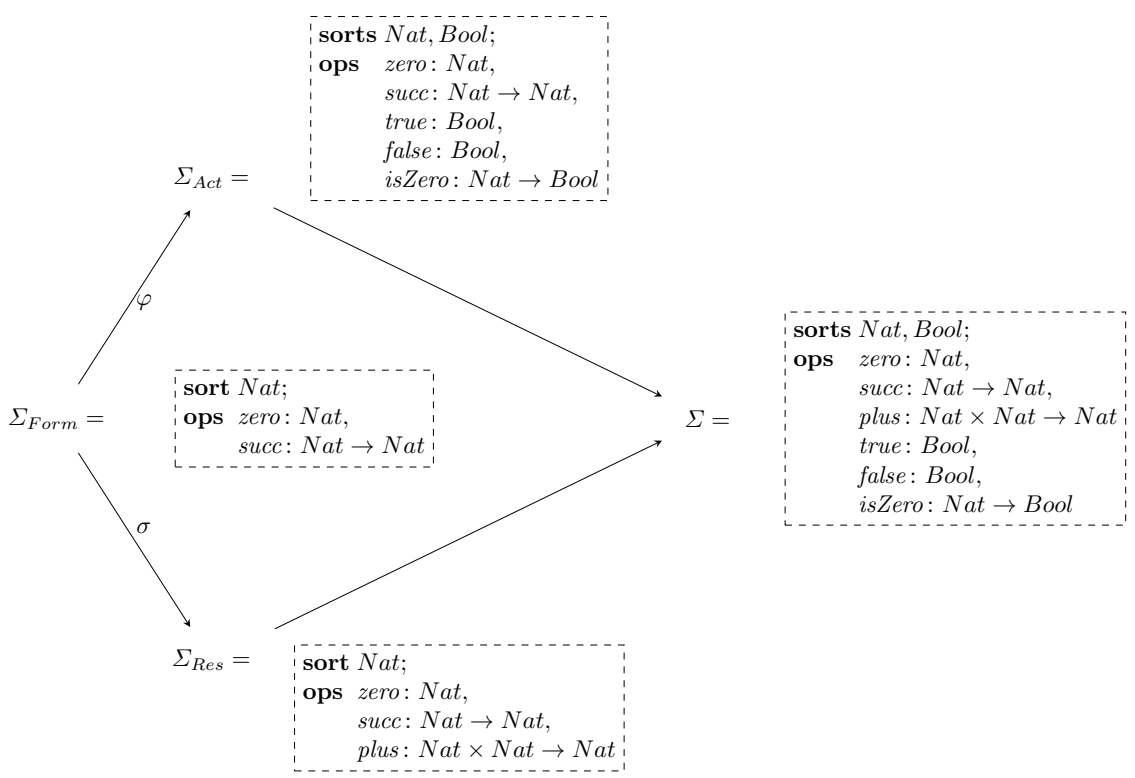

The construction signature $\sigma$ defines an operation plus: Nat $\times$ Nat $\rightarrow$ Nat, provided it is given a sort Nat with operations zero: Nat and succ: Nat $\rightarrow$ Nat. The actual parameters signature $\Sigma_{A c t}$ enriches the formal parameters signature $\Sigma_{F o r m}$ by several symbols like Bool, true: Bool etc. The pushout signature contains all symbols together. However, the information about a potential dependency of the operation plus on Nat, zero, succ is lost.

Here comes the idea to enrich the signatures by dependency structure to explicitly show how symbols depend on other symbols. The diagram below shows how we imagine the dependency. It shall be a transitive relation (depicted as dashed lines) on set of symbols taken from the signature.

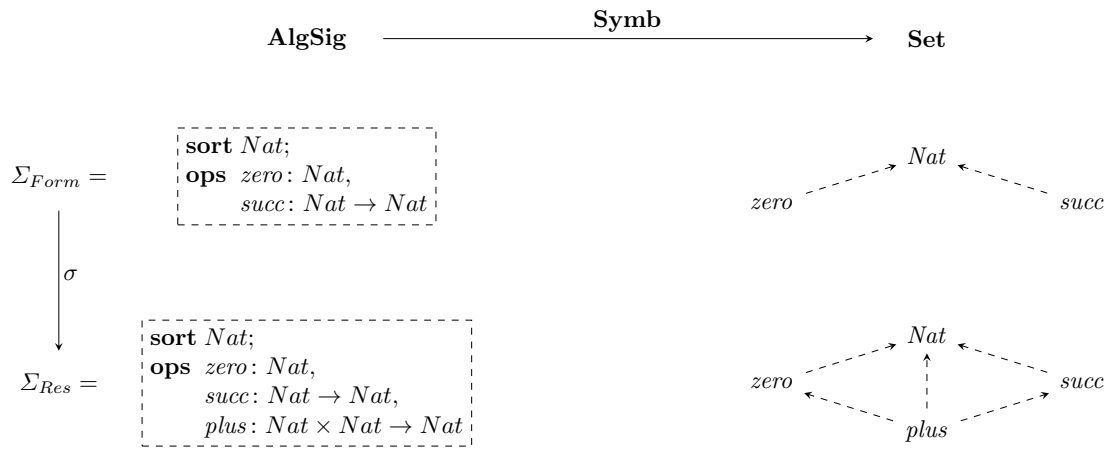


In the pushout, the dependencies should be preserved, as on the following diagram. It is visible that the operation isZero doesn't depend on plus. Neither the later depends on the former.

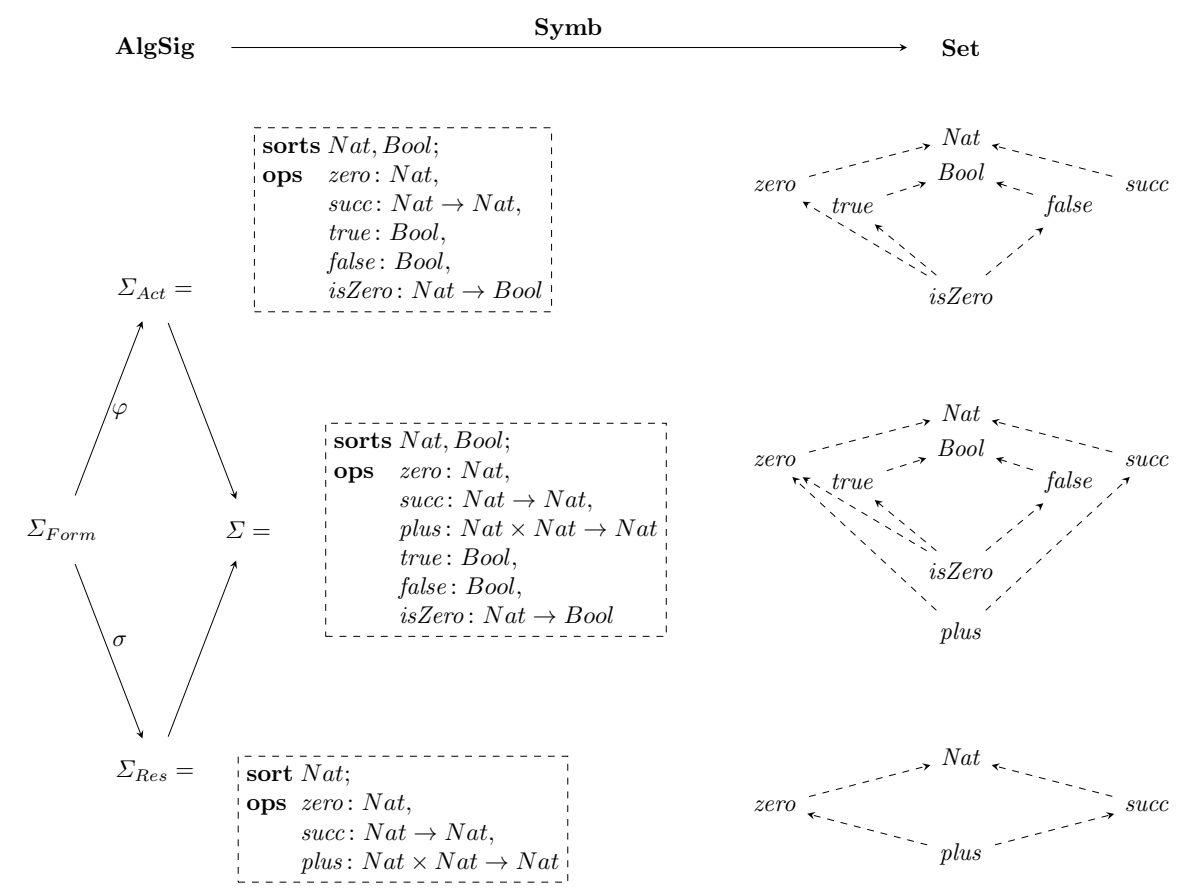

In our paper we try to answer the following questions.

1. What kind of dependency relation shall we use?

2. How to enrich the signatures by dependency structure?

3. How to define morphism between such enriched signatures?

The simple example given above already says something about the dependency relation - it needs to be transitive. We also have to make sure that our extension of signatures is defined as a category that keeps all important properties of the original category AlgSig. Namely, we require that morphisms shouldn't change (in)dependencies of symbols and that the category of enriched signatures have all pushouts and pullbacks.

\section{Dependency relation}

We start our work by investigating properties of several categories of sets ordered by various transitive relations. We consider morphisms that not only preserve the dependencies, but also weakly reflect their structure. 


\subsection{Category Rset $\downarrow$ and its Subcategories}

As we already mentioned in the previous section, we consider only transitive relations as candidates for the dependency relation.

Definition 1 (R-sets) An R-set ${ }^{1}$ is a pair $\left\langle A, R_{A}\right\rangle$ where $R_{A} \subseteq A^{2}$ is a transitive relation on a set $A$. In what follows we sometimes write $A_{R}$ instead of $\left\langle A, R_{A}\right\rangle$. We may use the infix notation for $R_{A}$ and for $a_{1}, a_{2} \in A_{R}$ we may also write $a_{1} R a_{2}$ instead of $a_{1} R_{A} a_{2}$, when decorations are clear from the context.

Definition 2 (Category Rset $\downarrow$ of R-sets and P-morphisms) A category $\mathbf{R s e t} \downarrow$ has $\mathrm{R}$-sets as objects and pseudo-epimorphisms, or p-morphisms, as morphisms. $A$ p-morphism is a function that preserves the relation $R$ and weakly reflects $R$ set down-closures, i.e. a morphism $f:\left\langle A, R_{A}\right\rangle \rightarrow\left\langle B, R_{B}\right\rangle$ is a function $m: A \rightarrow$ $B$ such that:

1. (monotonicity) for all $a_{1}, a_{2} \in A, a_{1} R_{A} a_{2}$ implies $f\left(a_{1}\right) R_{B} f\left(a_{2}\right)$.

2. (weakly reflected $R$-down-closures) for all $a_{2} \in A, b_{1} \in B, b_{1} R_{B} f\left(a_{2}\right)$ implies that there exists $a_{1} \in A$, that $a_{1} R_{A} a_{2}$ and $f\left(a_{1}\right)=b_{1}$.

Identities and composition are defined as expected.

Note that p-morphisms are such functions between ordered sets, that their graph is a bisimulation of orders seen as transitive systems.

Definition 3 (Sub R-set) Given an $R$-set $A_{R}$ and $a \in A$, its closed down sub $\mathrm{R}$-set induced by an element $a$ is defined as:

$$
A_{R}{ }^{a} \downarrow=A_{R}^{\prime}
$$

where $A^{\prime}=\left\{a^{\prime} \in A \mid a^{\prime} R_{A} a\right\} \cup\{a\}$ and $R_{A^{\prime}}=\left.R_{A}\right|_{A^{\prime}}$.

It is important to notice that $a \in A_{R}{ }^{a} \downarrow$, for any set $A, a \in A$ and a relation $R$.

Below we formalize several full subcategories of the category Rset $\downarrow$. We think that they may be good candidates for the signature-symbols dependency orderings. In the following sections we are going to investigate their properties to choose the most appropriate one.

Definition 4 (Category Preord $\downarrow$ ) A category Preord $\downarrow$ of preorders and $p$ morphisms is a full subcategory of Rset $\downarrow$ where objects are preorders: an R-set $\left\langle A, \leq_{A}\right\rangle$ is a Preord $\downarrow$-object iff the relation $\leq_{A}$ is transitive and reflexive.

Definition 5 (Category Soset $\downarrow$ ) A category Soset $\downarrow$ of strict orders and pmorphisms is a full subcategory of Rset $\downarrow$ where objects are strict orders, i.e. an $R$-set $\left\langle A,<_{A}\right\rangle$ is a Soset $\downarrow$-object iff the relation $<_{A}$ is transitive and asymmetric; thus, irreflexive.

\footnotetext{
${ }^{1}$ In the model-theory the same mathematical structure is called a transitive Kripke frame [Seg70] thus, all results presented in this paper also concern transitive Kripke frames. The author would like to thank Bartek Klin for pointing out that fact.
} 
Definition 6 (Category Soset ( $_{\mathrm{wf}} \downarrow$ ) A category Soset $_{\mathrm{w}} \downarrow \downarrow$ of well-founded strict orders and p-morphisms is a full subcategory of Soset $\downarrow$. Its objects are wellfounded strict orders, i.e. strict orders without infinite descending chains.

To safe space we don't discuss the possibility to have partial orders as a candidate for dependency relation. We checked that they are unacceptable.

\section{$3.2 \quad$ R-multisets and Dependency Bisimulation}

This section defines R-multisets that become handy when it comes to definition of products in the category Rset $\downarrow$ and its subcategories (cf. Sect. 4). The reader may skip this section in the first reading.

The idea is to take an R-set and define a multiset of its elements without adding new dependencies; however, some original dependencies may be dropped.

Definition 7 (Labelled R-set) $A$ labeled R-set is a triple $\left\langle A_{R}, P_{R}, \mu\right\rangle$ where $A_{R}$ and $P_{R}$ are $R$-sets and $\mu: A_{R} \rightarrow P_{R}$ is a monotone labeling function.

Definition 8 (Labelled R-set Isomorphism) Two labeled $R$-sets $\left\langle A_{R}, P_{R}, \mu\right\rangle$, $\left\langle A_{R}^{\prime}, P_{R}, \mu^{\prime}\right\rangle$ are isomorphic iff there exists a bijection $\tau: A \rightarrow A^{\prime}$ such that for all $a \in A, \mu(a)=\mu^{\prime}(\tau(a))$ and for all $a, a^{\prime} \in A$ a $R_{A} a^{\prime}$ iff $\tau(a) R_{A^{\prime}} \tau(a)$.

Definition 9 (R-mset - R-multiset) An R-multiset, or R-mset, $\left[A_{R}, P_{R}, \mu\right]$ is the isomorphism class ${ }^{2}$ of a labeled $R$-set $\left\langle A_{R}, P_{R}, \mu\right\rangle$.

Definition 10 (R-submultiset) Given an $R$-mset $\left[A_{R}, P_{R}, \mu\right]$ and $a \in A$, its R-submultiset $\left[A_{R}, P_{R}, \mu\right]^{a} \downarrow$ induced by $a$ is defined as an $R$-mset:

$$
\left.\left[A_{R}, P_{R}, \mu\right]^{a} \downarrow=\left[A_{R}^{a} \downarrow, P_{R},\left.\mu\right|_{\left(A_{R}{ }^{a} \downarrow\right.}\right)\right]
$$

Lemma 11 Given an $R$-mset $\left[A_{R}, P_{R}, \mu\right]$ and $a, a^{\prime} \in A$, such that $a^{\prime} R a$, it holds that

$$
\left[A_{R}, P_{R}, \mu\right]^{a^{\prime}} \downarrow=\left(\left[A_{R}, P_{R}, \mu\right]^{a} \downarrow\right)^{a^{\prime}} \downarrow
$$

Having a regular multiset we can easily calculate the set of its distinct elements. The similar question can be asked with regard to R-multisets, but here the matter is to find a R-multiset of elements that have the distinct dependency structure. In the following definition we use the bisimulation to find the kernel of the given R-mset. It is going to play a crucial role in the definition of products in the category Rset $\downarrow$ and its subcategories.

Definition 12 (Kernel Relation of R-mset) Given an $R$-mset $\left[A_{R}, P_{R}, \mu\right]$ its kernel relation $K\left(\left[A_{R}, P_{R}, \mu\right]\right) \subseteq A^{2}$ is the greatest dependency bisimulation

\footnotetext{
${ }^{2}$ For technical convenience we will sometimes define concepts and constructions on R-msets by introducing them on representatives - leaving to the Reader the details of generalization to their isomorphism classes.
} 
relation on $A$. A dependency bisimulation is an equivalence relation $\sim \subseteq A^{2}$, such that for $a_{1}, a_{2} \in A$

$$
\begin{aligned}
& \text { if } a_{1} \sim a_{2} \text { then } \mu\left(a_{1}\right)=\mu\left(a_{2}\right) \text { and } \\
& \qquad \begin{array}{l}
\text { for all } a_{1}^{\prime} \in A \text { such that } a_{1}^{\prime} R a_{1} \\
\text { there exists } a_{2}^{\prime} \in A, a_{2}^{\prime} R a_{2}, a_{1}^{\prime} \sim a_{2}^{\prime} \\
\text { and for all } a_{2}^{\prime} \in A \text { such that } a_{2}^{\prime} R a_{2} \\
\text { there exists } a_{1}^{\prime} \in A, a_{1}^{\prime} R a_{1}, a_{1}^{\prime} \sim a_{2}^{\prime}
\end{array}
\end{aligned}
$$

The family of dependency bisimulations is non-empty (it contains $i d_{A}$ ) and it is closed under the unions, hence the kernel relation exists for every $R$-mset $\left[A_{R}, P_{R}, \mu\right]$.

\section{Limits in Rset $\downarrow$}

In this section we try to find out whether the categories from Sect. 3.1 have enough limits. We are particularly interested in the existence of pullbacks, which we are going to define through equalizers and products of nonempty families of objects. However, to have a complete picture, we also look at the existence of final objects.

Theorem 13 The category Preord $\downarrow$ has a singleton ordered by identity as its final object.

Theorem 14 The categories Soset $\downarrow$ and Soset $_{\mathbf{w f}} \downarrow$ do not have a final object.

Proof: Since the relations in objects of Soset $\downarrow$ (and Soset $_{\mathbf{w f}} \downarrow$ ) are irreflexive, their morphisms must not glue together any elements being in relation. Hence, if the final object existed, there would be an injective map from any ordinal (represented as an R-set with natural strict well-founded "ordering") into it. Hence, such a final object can not be a proper set.

Conjecture 15 The category $\mathbf{R s e t} \downarrow$ does not have a final object.

Only the category Preord $\downarrow$ proves to have the final object, but the lack of it does not disqualify the others. Importantly, we can prove that all above-defined categories have the equalizers.

Theorem 16 The category Rset $\downarrow$ and its subcategories Preord $\downarrow$, Soset $\downarrow$ and Soset $_{\mathbf{w f} \downarrow} \downarrow$ have all equalizers.

The equalizers are the same as in Set. For the proof see App. A of [Mar10].

Now, we come to the most tricky part of the paper, namely products in R-sets. At first their definition seem obvious, but the deeper look unveils the quite surprising setting. Let us show it by an example in Preord $\downarrow$. Let $A_{\leq}=$ 
$\left\langle\left\{a_{1}, a_{2}\right\}, a_{1} \leq a_{1}, a_{2} \leq a_{2}, a_{2} \leq a_{1}\right\rangle$ and $B_{\leq}=\left\langle\left\{b_{1}, b_{2}\right\}, b_{1} \leq b_{1}, b_{2} \leq b_{2}, b_{2} \leq b_{1}\right\rangle$ be two Preord $\downarrow$-objects. Their product of $A_{\leq}$and $B_{\leq}$in Preord $\downarrow$ is $Q_{\leq}$that may be depicted as below.

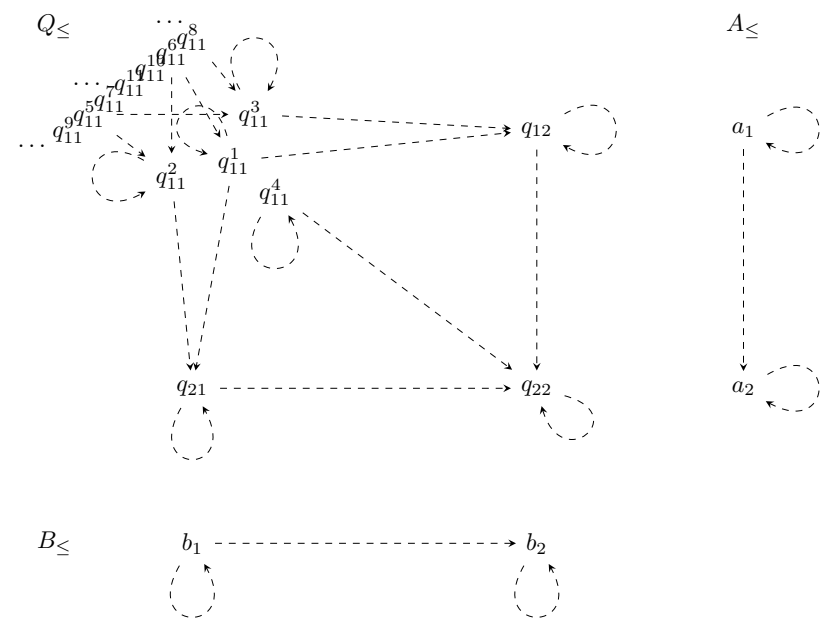

As expected there are $q_{22}=\left\langle a_{2}, b_{2}\right\rangle, q_{12}=\left\langle a_{1}, b_{2}\right\rangle, q_{21}=\left\langle a_{2}, b_{1}\right\rangle$ in $Q_{\leq}$. They are in relation with themselves and also $q_{22} \leq_{Q} q_{21}$ and $q_{22} \leq_{Q} q_{12}$. There is also $q_{11}^{1}=\left\langle a_{1}, b_{1}\right\rangle$ that is in relation with all above mentioned elements. However, there are also infinity many distinct elements of $Q_{\leq}$, also, as $q_{11}^{1}$ projected to $a_{1}$ and $b_{1}$, marked above as $q_{11}^{2}, q_{11}^{3}, \ldots$ that differ on their dependency relations.

As we are going to prove later in Lemma 18, the following definition proposes the construction of the product. The only problem is that in some cases the structure it describes may fail to be a set.

Definition 17 (Product Candidate) Given two Rset $\downarrow$-objects $\left\langle A, R_{A}\right\rangle$ and $\left\langle B, R_{B}\right\rangle$, let us define a product candidate as a pair of a class and a relation $\left\langle A \prod B, R_{A \prod B}\right\rangle$ together with two functions $\rho_{A}: A \prod B \rightarrow A$ and $\rho_{B}: A \prod B \rightarrow B$. In Lemma 18 we prove that whenever $A \prod B$ happens to be a set, then $\left\langle A \prod B, R_{A \prod B}\right\rangle$ with projections $\rho_{A}$ and $\rho_{B}$ is a product of the given $\mathbf{R s e t} \downarrow$-objects.

First, let $\left\langle P, R_{P}\right\rangle$ be a pair of the set $P=A \times B$, the product of $A$ and $B$ in Set together with projections $\pi_{A}: P \rightarrow A$ and $\pi_{B}: P \rightarrow B$, and a relation $R_{P} \subseteq P^{2}:$

$$
R_{P}=\left\{\left\langle p_{1}, p_{2}\right\rangle \in P^{2} \mid \pi_{A}\left(p_{1}\right) R_{A} \pi_{A}\left(p_{2}\right) \text { and } \pi_{B}\left(p_{1}\right) R_{B} \pi_{B}\left(p_{2}\right)\right\}
$$


Now, let the class ${ }^{3} A \prod B$ be defined as:

$$
\begin{aligned}
A \prod^{B=}=\bigcup_{p \in P}\left\{\left\langle\left[X_{R}, P_{R}, \mu\right], p\right\rangle \mid\right. & \\
& {\left[X_{R}, P_{R}, \mu\right] \text { is an R-mset such that: } } \\
& \bullet K\left(\left[X_{R}, P_{R}, \mu\right]\right)=i d_{X}, \\
& \bullet \mu ; \pi_{A} \text { and } \mu ; \pi_{B} \text { are morphisms in } \mathbf{R s e t} \downarrow, \\
& \bullet \text { there exists } x \in X, \text { such that } \mu(x)=p \\
& \text { and for all } x^{\prime} \in X, \text { if } x^{\prime} \neq x \text { then } x^{\prime} R_{X} x \\
\} &
\end{aligned}
$$

See Def. 9, 12 for the definition of $R$-msets and their kernels. The relation $R_{A \prod B}$ is defined as follows:

$$
\begin{gathered}
R_{A \prod B}=\left\{\left\langle\left\langle\left[X_{R}^{\prime}, P_{R}, \mu^{\prime}\right], p^{\prime}\right\rangle,\left\langle\left[X_{R}, P_{R}, \mu\right], p\right\rangle\right\rangle \in\left(A \prod B\right)^{2} \mid\right. \\
\text { there exists } x^{\prime} \in X, \mu\left(x^{\prime}\right)=p^{\prime}, p^{\prime} R p \\
\left.\quad \text { and }\left[X_{R}^{\prime}, P_{R}, \mu^{\prime}\right]=\left[X_{R}, P_{R}, \mu\right]^{x^{\prime}} \downarrow\right\}
\end{gathered}
$$

Cf. Def. 10 for the definition of $R$-submultisets.

We define product-candidate projection functions $\rho_{A}:\left(A \prod B\right) \rightarrow A$ and $\rho_{B}:\left(A \prod B\right) \rightarrow$ $B$ as

$$
\begin{aligned}
& \rho_{A}\left(\left\langle\left[X_{R}, P_{R}, \mu\right], p\right\rangle\right)=\pi_{A}(p) \\
& \rho_{B}\left(\left\langle\left[X_{R}, P_{R}, \mu\right], p\right\rangle\right)=\pi_{B}(p)
\end{aligned}
$$

The class $A \prod B$ contains every element of $P$ taken as many times, as there are distinct (wrt. the kernel relation) R-msets of elements lower than it wrt. $R_{P}$. These R-msets are subject to the requirement that their labellings composed with Cartesian product projections are p-morphisms. This makes them weakly reflect the R-down-closures of $A_{R}$ and $B_{R}$.

The following lemma guarantees that once we show that the product candidate is a set, then it is indeed the product.

Lemma 18 Consider two Rset $\downarrow$-objects $A_{R}$ and $B_{R}$, if $A \prod B$ is a set, then the product candidate $\left\langle A_{R} \prod B_{R}, R_{A} \prod_{B}\right\rangle$ is their product in Rset $\downarrow$.

The proof is quite technical (see App. A of [Mar10]). The main point is to observe that when there is any other object with two projections to $A_{R}$ and $B_{R}$, it may be seen as an R-multiset. We take its kernel (cf. Def. 12) and find that every element of the kernel (with its dependency structure) is also present in $A \prod B$. It allows us to define the unique morphism from the given object to $A \prod B$.

The products of $n$ R-sets, for $n>2$, if they exists, are defined following the same idea.

\footnotetext{
${ }^{3}$ In general, for a given nonempty set of labels $P$, the class of all $P$-labeled R-msets is a proper class.
} 
Lemma 19 Given two Rset $\downarrow$-objects $\left\langle A, A_{R}\right\rangle$ and $\left\langle B, R_{B}\right\rangle$, if both $R_{A}$ and $R_{B}$ are reflexive / irreflexive / asymmetric / strict well-founded, then the relation $R_{A \prod \text { B }}$ from Def. 17 is also so.

The proof is straightforward. For details see App. A of [Mar10].

Theorem 20 The category Soset $_{\mathrm{wf}} \downarrow$ has all binary products. Moreover, given two Soset $_{\mathbf{w f}} \downarrow$-objects, $A_{<}, B_{<}$, their product is isomorphic to the product candidate from Def. 17.

We prove that, given any $A_{<}, B_{<} \in$ Soset $_{\mathbf{w f}} \downarrow$, the product candidate $A \prod B$ is a set. Then, by Lemma 18 , we argue that $\left(A \prod B\right)_{<}$is indeed the product of the given objects. The proof goes by induction. We bound the number of possible distinct structures labeled by every $p \in P_{<}$. Since dependency relations in question are well-founded, this is possible to do so. For details see App. A of [Mar10].

We cannot find the similar proof for Rset $\downarrow$, Preord $\downarrow$ and Soset $\downarrow$, hence the following conjecture.

Conjecture 21 The category Rset $\downarrow$ and its subcategories Preord $\downarrow$ and Soset $\downarrow$ do not have all binary products.

\section{Colimits in Rset $\downarrow$}

After the struggle with the limits in Rset $\downarrow$ and its subcategories, colimits are easy and boring. Basically the colimits in these categories are the same as in Set. The only exception is Soset $\downarrow$, which does not have all coequalizers.

Lemma 22 Given two Rset $\downarrow$-morphisms $f, g: A_{R} \rightarrow B_{R}$, their coequalizer in Set, $e: B \rightarrow C$, is an Rset $\downarrow$-morphism e: $B_{R} \rightarrow C_{R}$ where the relation $R_{C}$ is defined simply as

$$
R_{C}=e\left(R_{B}\right)
$$

Theorem 23 The category Rset $\downarrow$ and its subcategory Preord $\downarrow$ have all coequalizers.

In case of Rset $\downarrow$ the proof is just a straight use of Lemma 22. For Preord $\downarrow$ we show that $e$ preserves the reflexivity of $R_{B}$ (cf. App. A of [Mar10]). However, the irreflexivity of $R_{B}$ does not cause the irreflexivity of $e\left(R_{B}\right)$, hence the following theorem.

Theorem 24 The category Soset $\downarrow$ does not have all coequalizers.

The counterexample involves two functions $i d: \mathbb{N} \rightarrow \mathbb{N}$ and succ: $\mathbb{N} \rightarrow \mathbb{N}$ and the inverse ordering of natural numbers. See App. A of [Mar10] for details.

It is enough to limit strict orders to well-founded orders to avoid counterexamples like the one presented above. 
Theorem 25 The category Soset $_{\mathrm{wf}} \downarrow$ has all coequalizers.

The App. A of [Mar10] contains the proof. Finally, the easy part concerns coproducts.

Theorem 26 The category Rset $\downarrow$ and its subcategories Preord $\downarrow$,Soset $\downarrow$ and Soset $_{\mathbf{w}} \downarrow \downarrow$ have all coproducts

The coproducts are same as in Set.

\section{Algebraic Signatures with Dependent Symbols}

The two previous sections left only one ordering suitable for a dependency relation, namely well-founded strict ordering category $\operatorname{Soset}_{\mathbf{w f}} \downarrow$. All others probably don't have all products and Soset $\downarrow$ does not have coequalizers.

In this section we formalize what we hand-waved in Sect. 2. We define the category of algebraic signatures and enrich it by the dependency structure.

Definition 27 (Algebraic Signatures) We define a category AlgSig in the standard way - with objects being algebraic signatures defined as pairs of the form $\Sigma=\left\langle S, \Omega_{S^{+}}\right\rangle$where $S \in$ Set is a set of sorts, $S^{+}$is a set of nonempty finite $S$-sequences and $\Omega_{S^{+}}=\left\langle\Omega_{e}\right\rangle_{e \in S^{+}}$is an $S^{+}$-sorted set of operation names. Morphisms of AlgSig are pairs of the form $\left\langle\sigma_{S}, \sigma_{\Omega_{S}}\right\rangle: \Sigma \rightarrow \Sigma^{\prime}$ where $\sigma_{S}: S \rightarrow$ $S^{\prime}$ and $\sigma_{\Omega_{S}}=\left\langle\sigma_{\Omega_{e}}: \Omega_{e} \rightarrow \Omega_{\sigma_{S}^{+}(e)}^{\prime}\right\rangle_{e \in S^{+}}$. Identities and composition are defined as one may expect.

Before we allow a general dependency structure o symbols, we define a functor that recognizes the basic dependency of operation symbols on sort symbols, as discussed in Sect. 2.

Definition 28 (SigSymb Functor) Let SigSymb: AlgSig $\rightarrow$ Soset $_{\mathbf{w f}} \downarrow$ be the functor that transforms algebraic signatures to well-founded strict orders of signatures' symbols. Given an algebraic signature $\Sigma=\left\langle S, \Omega_{S^{+}}\right\rangle$we define

$$
\operatorname{SigSymb}(\Sigma)=\left\langle s \uplus\left(\biguplus_{e \in S^{+}}\left\{o: e \mid o \in \Omega_{e}\right\}\right),<\operatorname{SigSymb}(\Sigma)\right\rangle
$$

having operation symbols naturally dependent on sorts of their result and from their arities; i.e. for all $e \in S^{+}, e=\left\langle s_{0} \ldots s_{n}\right\rangle, o \in \Omega_{e}$, we have

$$
\left(s_{k}\right)<\operatorname{SigSymb}(\Sigma)(o: e)
$$

for all $0<k<n$. Given an algebraic signature morphism $\sigma=\left\langle\sigma_{s}, \sigma_{\Omega_{S}}\right\rangle: \Sigma \rightarrow$ $\Sigma^{\prime}$, we define a $\operatorname{Soset}_{\mathbf{w f} \downarrow} \downarrow-m o r p h i s m \operatorname{Sig} \operatorname{Symb}(\sigma): \operatorname{SigSymb}(\Sigma) \rightarrow \operatorname{SigSymb}\left(\Sigma^{\prime}\right)$ as

$$
\operatorname{SigSymb}(\sigma)=\sigma_{s} \uplus\left(\biguplus_{e \in S^{+}} \sigma_{\Omega_{e}}^{\prime}\right)
$$

Where $\sigma_{\Omega_{e}}^{\prime}(o: e)=\sigma_{\Omega_{e}}(o)$ for $o \in \Omega_{e}$. By construction it meets both requirements from Def. 2. 
Of course the dependency may be forgotten, if one wishes.

Definition 29 (SetSymb Functor) Let a functor that gives a set of signature's symbols, SetSymb: AlgSig $\rightarrow$ Set, be defined as SetSymb = SigSymb; $\mathbf{U}_{\text {Soset }_{\mathbf{w}}}$ where $\mathbf{U}_{\mathbf{S o s e t}_{\mathbf{w}}}$ : Soset $_{\mathbf{w f}} \downarrow \rightarrow$ Set is the obvious forgetful functor.

At the moment we have everything that we need to define the structures from the paper's title.

Definition 30 (Algebraic Signatures with Dependent Symbols) Objects of a category AlgSigDep of algebraic signatures with dependent symbols are pairs

$$
\Sigma_{<}=\left\langle\Sigma,<_{\Sigma}\right\rangle
$$

where $\Sigma \in \mathbf{A l g S i g}$ is an algebraic signature and $<_{\Sigma} \subseteq \operatorname{SigSymb}(\Sigma) \times \operatorname{SigSymb}(\Sigma)$ is such dependency relation that $<_{\operatorname{SigSymb}(\Sigma) \subseteq<_{\Sigma}}($ cf. Def. 28) and

$$
\left\langle\operatorname{SetSymb}(\Sigma),<_{\Sigma}\right\rangle \in \operatorname{Soset}_{\mathbf{w f} \downarrow}
$$

Morphisms between algebraic signatures with dependent symbols $\Sigma_{<}, \Sigma_{<}^{\prime} \in \mathbf{A l g S i g D e p}$ are such algebraic signature morphisms $\sigma: \Sigma \rightarrow \Sigma^{\prime}$, for which a function $\mathbf{S i g S y m b}(\sigma)$ seen as a morphism $\operatorname{SigSymb}(\sigma):\left\langle\operatorname{Set} \operatorname{Symb}(\Sigma),<_{\Sigma}\right\rangle \rightarrow\left\langle\operatorname{SetSymb}\left(\Sigma^{\prime}\right),<_{\Sigma^{\prime}}\right\rangle$

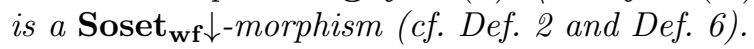

Till the end of this section we define functors and present lemmas needed in the following sections.

Definition 31 (DepSymb Functor) A functor DepSymb: AlgSigDep $\rightarrow$ Soset $_{\mathbf{w f}} \downarrow$ is defined as $\operatorname{DepSymb}\left(\Sigma_{<}\right)=\left\langle\operatorname{SetSymb}(\Sigma),<_{\Sigma}\right\rangle$, for a signature with dependent symbols $\Sigma_{<} \in \mathbf{A l g S i g D e p , ~ a n d ~} \operatorname{DepSymb}(\sigma)=\operatorname{SetSymb}(\sigma)$, for a signature morphism $\sigma \in$ AlgSigDep.

Definition 32 (Symb Functor) Let a functor that gives a set of symbols of the signature with dependent symbols, Symb: AlgSigDep $\rightarrow$ Set, be defined as $\mathbf{S y m b}=$ DepSymb; $\mathbf{U}_{\text {Soset }_{\mathbf{w}} \downarrow}$ where again $\mathbf{U}_{\text {Soset }_{\mathbf{w}}}$ : Soset $_{\mathbf{w f} \downarrow} \downarrow \rightarrow$ Set is the obvious forgetful functor.

Lemma 33 The functor Symb: AlgSigDep $\rightarrow$ Set is faithful.

Definition 34 (Embedding AlgSig into AlgSigDep) Category of algebraic signatures is naturally embeddable into the category of algebraic signatures with dependent symbols via the functor Dep: AlgSig $\rightarrow$ AlgSigDep defined as $\operatorname{Dep}(\Sigma)=\left\langle\Sigma,<_{\operatorname{DepSymb}(\Sigma)}\right\rangle$, for a signature $\Sigma \in \mathbf{A l g S i g}$, and $\operatorname{Dep}(\sigma)=\sigma$, for a signature morphism $\sigma \in \mathbf{A l g S i g . ~}$

Lemma 35 It holds that SetSymb = Dep; Symb. 


\subsection{Reconstructing Signatures with Dependent Symbols}

Converting a signature to a set of symbols should be complemented by an inverse operation. However, we can not just in an ad-hoc manner add a signature's structure to a given set. Instead, we propose a signature "reconstruction" given a function from an arbitrary set to an (ordered) set of signature's symbols.

Definition 36 (Signature Reconstruction) Let us define a signature with dependent symbols-reconstruction functor

$$
\text { Rec: }(\text { Set } \downarrow \text { Symb) } \rightarrow \text { AlgSigDep }
$$

where (Set $\downarrow$ Symb) is a comma category.

- Given a (Set $\downarrow$ Symb)-object, i.e. a function $f: A \rightarrow \operatorname{Symb}\left(\Sigma_{<}\right)$, for some AlgSigDep-signature $\Sigma_{<}=\left\langle\left\langle S, \Omega_{S^{+}}\right\rangle,<_{\Sigma}\right\rangle$ and some set $A \in$ Set, we "reconstruct" the greatest signature derived from $\Sigma_{<}$wrt. $f$ that has symbols from $A$

$$
\boldsymbol{\operatorname { R e c }}(f)=\left\langle\left\langle S^{A}, \Omega_{S^{A+}}^{A}\right\rangle,<_{\boldsymbol{R e c}(f)}\right\rangle
$$

where $S^{A}=\left\{s \in A^{\prime} \mid f(s) \in S\right\}$ and for any $e \in S^{A^{+}}, \Omega_{e}^{A}=\{o: e \mid$ $o \in A^{\prime}$ and $\left.f(o) \in \Omega_{f(e)}\right\}$ and $A^{\prime}=\left\{a \in A \mid f(A)_{<\Sigma}{ }^{f(a)} \downarrow \subseteq f(A)\right\}$ and $<_{\boldsymbol{R e c}(f)}=\left(<_{\Sigma} \mid f\right) \|_{A^{\prime}}$

- For a (Set $\downarrow$ Symb)-morphism, i.e. a pair of morphisms $\left\langle g: A_{1} \rightarrow A_{2}, \sigma: \Sigma_{<}^{1} \rightarrow \Sigma_{<}^{2}\right\rangle$ where $\sigma$ is AlgSigDep-morphism and $g$ is a function such that $g ; f_{2}=$ $f_{1} ; \operatorname{Symb}(\sigma)$ and $f_{i}: A_{i} \rightarrow \operatorname{Symb}\left(\Sigma_{<}^{i}\right)$, for $i \in\{1,2\}$, are two (Set $\downarrow$ Symb)-objects, a AlgSigDep-morphism between "reconstructed" signatures is defined as

$$
\boldsymbol{\operatorname { R e c }}(\langle g, \sigma\rangle)=\left\langle\sigma_{S}^{\prime}, \sigma_{\Omega_{S} A_{1}}^{\prime}\right\rangle: \operatorname{Rec}\left(f_{1}\right) \rightarrow \operatorname{Rec}\left(f_{2}\right)
$$

where $\sigma_{S}^{\prime}=g_{S^{A_{1}}}$ and for every $e \in S^{A_{1}+}, \sigma_{\Omega_{e}}^{\prime}=g_{\Omega_{e}^{A_{1}}}$.

Note that not all symbols from a given set stays in the "reconstructed" signature - only these that meet the dependency compatibility conditions. The following definition unveils exactly what kind of inverse is Rec to Symb. The subsequent lemma proves that they are locally adjoint.

Definition 37 For every signature with dependent symbols, $\Sigma_{<}=\left\langle S, \Omega_{S^{+}}\right\rangle \in$ AlgSigDep, there exist the following two functors. The under- $\Sigma_{<}$"symbol" functor

$$
\operatorname{Symb}_{\Sigma_{<}}:\left(\operatorname{AlgSigDep} \downarrow \Sigma_{<}\right) \rightarrow\left(\operatorname{Set} \downarrow \operatorname{Symb}\left(\Sigma_{<}\right)\right)
$$

defined

- for an (AlgSigDep $\downarrow \Sigma_{<}$)-object $\sigma: \Sigma_{<}^{\prime} \rightarrow \Sigma_{<}$as

$$
\operatorname{Symb}_{\Sigma_{<}}(\sigma)=\operatorname{Symb}(\sigma): \operatorname{Symb}\left(\Sigma_{<}^{\prime}\right) \rightarrow \operatorname{Symb}\left(\Sigma_{<}\right)
$$


- for an (AlgSigDep $\left.\downarrow \Sigma_{<}\right)$-morphism $\varphi: \Sigma_{<}^{1} \rightarrow \Sigma_{<}^{2}$ as

$$
\operatorname{Symb}_{\Sigma_{<}}(\varphi)=\operatorname{Symb}(\varphi): \operatorname{Symb}\left(\Sigma_{<}^{1}\right) \rightarrow \operatorname{Symb}\left(\Sigma_{<}^{2}\right)
$$

and the under- $\Sigma_{<}$"reconstruction" functor

$$
\operatorname{Rec}_{\Sigma_{<}}:\left(\operatorname{Set} \downarrow \operatorname{Symb}\left(\Sigma_{<}\right)\right) \rightarrow\left(\operatorname{AlgSigDep} \downarrow \Sigma_{<}\right)
$$

defined

- for an $\left(\operatorname{Set} \downarrow \operatorname{Symb}\left(\Sigma_{<}\right)\right)$-object $f: A \rightarrow \operatorname{Symb}\left(\Sigma_{<}\right)$as

$$
\operatorname{Rec}_{\Sigma_{<}}(f)=\left\langle\varphi_{S}, \varphi_{\Omega_{S}}\right\rangle: \operatorname{Rec}(f) \rightarrow \Sigma_{<}
$$

where $\operatorname{Rec}(f)=\left\langle\left\langle S^{\prime}, \Omega_{S^{\prime}+}^{\prime}\right\rangle,<_{\boldsymbol{R e c}(f)}\right\rangle, \varphi_{S}=\left.f\right|_{S^{\prime}}$ and for every $e \in S^{\prime+}$, $\varphi_{\Omega_{e}}: \Omega_{e}^{\prime} \rightarrow \Omega_{\varphi_{S}^{+}(e)}$ is defined as $\varphi_{\Omega_{e}}(o)=f(o)$;

- for an $\left(\operatorname{Set} \downarrow \operatorname{Symb}\left(\Sigma_{<}\right)\right)$-morphism $g: A_{<}^{1} \rightarrow A_{<}^{2}$, with $f_{1}: A_{<}^{1} \rightarrow \operatorname{Symb}\left(\Sigma_{<}\right)$ and $f_{2}: A_{<}^{2} \rightarrow \operatorname{Symb}\left(\Sigma_{<}\right)$, as

$$
\boldsymbol{\operatorname { R e c }}_{\Sigma_{<}}(g)=\left\langle\gamma_{S}, \gamma_{\Omega_{S}}\right\rangle: \boldsymbol{\operatorname { R e c }}\left(f_{1}\right) \rightarrow \boldsymbol{\operatorname { R e c }}\left(f_{2}\right)
$$

where $\boldsymbol{\operatorname { R e c }}\left(f_{1}\right)=\left\langle\left\langle S_{1}, \Omega_{S_{1}+}^{1}\right\rangle,<_{\boldsymbol{R e c}\left(f_{1}\right)}\right\rangle, \gamma_{S}=g_{S_{1}}$ and for every $e \in S_{1}^{+}$, $\gamma_{\Omega_{e}}: \Omega^{1}{ }_{e} \rightarrow \Omega_{\gamma_{S}^{+}(e)}$ is defined as $\gamma_{\Omega_{e}}(o)=g(o)$

Lemma 38 For any $\Sigma_{<} \in$ AlgSigDep, the two functors from Def. 37 are adjoint in the following way

$$
\operatorname{Rec}_{\Sigma_{<}} \dashv \operatorname{Symb}_{\Sigma_{<}}
$$

Proof: Given a $\left(\operatorname{Set} \downarrow \operatorname{Symb}\left(\Sigma_{<}\right)\right)$-object $f: A \rightarrow \operatorname{Symb}\left(\Sigma_{<}\right)$and a $(\operatorname{Set} \downarrow$ $\operatorname{Symb}\left(\Sigma_{<}\right)$)-morphism $g: \operatorname{Symb}\left(\Sigma_{<}^{\prime}\right) \rightarrow A$, for some (AlgSigDep $\left.\downarrow \Sigma_{<}\right)$object $h: \Sigma_{<}^{\prime} \rightarrow \Sigma_{<}$, there exists exactly one (AlgSigDep $\downarrow \Sigma_{<}$)-morphism $g^{\#}: \Sigma_{<}^{\prime} \rightarrow \operatorname{Rec}(f)$ such that $\operatorname{Symb}\left(g^{\#}\right) ; \epsilon_{f}=g$, where $\epsilon_{f}$ is the counit function $\epsilon_{f}: \operatorname{Symb}(\boldsymbol{R e c}(f)) \rightarrow A$ naturally defined as $\epsilon_{f}(s)=s$ for $s \in S^{A}$ and $\epsilon_{f}(o:$ $e)=o$, for $o \in \Omega_{e}^{A}, e \in S^{A^{+}}$. To prove the existence of $g^{\#}$, let $\Sigma=\left\langle S, \Omega_{S^{+}}\right\rangle$and $\Sigma^{\prime}=\left\langle S^{\prime}, \Omega_{S^{\prime+}}^{\prime}\right\rangle$. The morphism $g^{\#}: \Sigma_{<}^{\prime} \rightarrow \boldsymbol{R e c}(f)$ is defined as $g^{\#}=\left\langle\sigma_{s}, \sigma_{\Omega_{S}}\right\rangle$, where $\sigma_{s}: S^{\prime} \rightarrow S$ and $\sigma_{\Omega_{S^{\prime}}^{\prime}}: \Omega_{S^{+}} \rightarrow \Omega_{S^{+}}$are given as: $\sigma_{s}(s)=g(s)$, for $s \in S^{\prime}$, $\sigma_{\Omega_{e}}(o)=g(o: e)$, for $e \in{S^{\prime}}^{+}$and $o \in \Omega_{e}^{\prime}$. By construction, the morphism $g^{\#}$ is the unique such that $\operatorname{Symb}\left(g^{\#}\right) ; \epsilon_{f}=g$.

Corollary 39 Given a function $f: A \rightarrow \operatorname{Symb}\left(\Sigma_{<}\right)$, it holds that

$$
\operatorname{Symb}\left(\boldsymbol{R e c}_{\Sigma_{<}}(f)\right)=\epsilon_{f} ; f
$$




\subsection{Limits and Colimits in AlgSigDep}

The rich technical background defined in the previous sections, regarding the signature symbols and their reconstructions, is to be used here to prove the existence of (co)limits in AlgSigDep.

Before we begin we would like to remind the reader that this is a standard result that the category AlgSig is both complete and cocomplete.

As in Sect. 4 we start our review of limits from the final object. Unfortunately it doesn't exist in AlgSigDep.

Theorem 40 The category AlgSigDep does not have the final object.

This is the straight consequence of the lack of the final object in Soset $_{\mathbf{w f}} \downarrow$. Fortunately all other (co)limits are present in this category

Theorem 41 The category AlgSigDep has all equalizers.

We use the Theorem 16 and the "reconstructing" functor from Def. 37. For the complete proof see App. A of [Mar10].

The construction of products in AlgSigDep follows these in Soset $_{\mathbf{w} f} \downarrow$. The result is then "reconstructed" to the form of a signature with dependency structure.

Theorem 42 The category AlgSigDep has all binary products.

Proof: The construction of the binary product in AlgSigDep follows the construction of a product candidate in Rset $\downarrow$ (cf. Def. 17).

Let $\Sigma_{<}^{A}$ and $\Sigma_{<}^{B}$ be two AlgSigDep-objects and let $\Sigma=\Sigma^{A} \times \Sigma^{B}$ and two projection morphism $\pi_{A}^{\Sigma}: \Sigma \rightarrow \Sigma^{A}$ and $\pi_{B}^{\Sigma}: \Sigma \rightarrow \Sigma^{B}$ be the product in AlgSig. Now, let $P=\operatorname{SetSymb}(\Sigma), \pi_{A}=\operatorname{SetSymb}\left(\pi_{A}^{\Sigma}\right), \pi_{B}=\operatorname{SetSymb}\left(\pi_{B}^{\Sigma}\right)$ and $<_{P}=\left\{\left\langle p_{1}, p_{2}\right\rangle \in P^{2} \mid \pi_{A}\left(p_{1}\right)<_{\Sigma_{A}} \pi_{A}\left(p_{2}\right)\right.$ and $\left.\pi_{B}\left(p_{1}\right)<\Sigma_{B} \pi_{B}\left(p_{2}\right)\right\}$.

Let a $\operatorname{set}^{4} Q$ be defined as:

$$
Q=\bigcup_{p \in P}\left\{\left\langle\left[X_{<}, P_{<}, \mu\right], p\right\rangle \mid\right.
$$

$\left[X_{<}, P_{<}, \mu\right]$ is a well founded strictly ordered R-mset such that:

- $K\left(\left[X_{<}, P_{<}, \mu\right]\right)=i d_{X}$,

- $\mu ; \pi_{A}$ and $\mu ; \pi_{B}$ are morphisms in Rset $\downarrow$,

- there exists $x \in X$, such that $\mu(x)=p$ and for all $x^{\prime} \in X$, if $x^{\prime} \neq x$ then $x^{\prime}<_{X} x$

\}

The well-founded strict order $<_{Q}$ is defined as follows:

$$
\begin{aligned}
<_{Q}=\{ & \left\{\left\langle\left[X_{<}^{\prime}, P_{<}, \mu^{\prime}\right], p^{\prime}\right\rangle,\left\langle\left[X_{<}, P_{<}, \mu\right], p\right\rangle\right\rangle \in Q^{2} \mid \\
& \text { there exists } x^{\prime} \in X, \mu\left(x^{\prime}\right)=p^{\prime}, p^{\prime}<p \\
& \text { and } \left.\left[X_{<}^{\prime}, P_{<}, \mu^{\prime}\right]=\left[X_{<}, P_{<}, \mu\right]^{x^{\prime}} \downarrow\right\}
\end{aligned}
$$

\footnotetext{
${ }^{4}$ Cf. Theorem 20 for the proof that a product candidate in $\operatorname{Soset}_{\mathbf{w f}} \downarrow$ is a set
} 
Let the top-element labeling function $l: Q \rightarrow P$ be defined as $l\left(\left\langle\left[X_{<}^{p}, P_{<}, \mu_{p}\right], p\right\rangle\right)=$ $p$. Let the projections be defined as $\rho_{A}=l ; \pi_{A}: Q_{<} \rightarrow \operatorname{DepSymb}\left(\Sigma_{<}^{A}\right)$ and $\rho_{B}=l ; \pi_{B}: Q_{<} \rightarrow \operatorname{DepSymb}\left(\Sigma_{<}^{B}\right)$.

Following the line of the proof of Lemma 18, the relation $<_{Q}$ is indeed a well-founded strict order and $\rho_{A}$ and $\rho_{B}$ are indeed Soset $_{\mathbf{w}} \downarrow \downarrow-$-morphisms.

The product of $\Sigma_{\leq}^{A}$ and $\Sigma_{\leq}^{B}$ in AlgSigDep is an AlgSigDep-object

$$
\Sigma_{<}^{Q}=\left\langle\boldsymbol{\operatorname { R e c }}(l),<_{Q} \mid \epsilon_{l}\right\rangle
$$

and two projection AlgSigDep-morphisms

$$
\begin{aligned}
& \rho_{A}^{\Sigma}=\operatorname{Rec}_{\operatorname{Dep}(\Sigma)}(l) ; \pi_{A}^{\Sigma}: \Sigma_{<}^{Q} \rightarrow \Sigma_{<}^{A} \\
& \rho_{B}^{\Sigma}=\operatorname{Rec}_{\operatorname{Dep}(\Sigma)}(l) ; \pi_{B}^{\Sigma}: \Sigma_{<}^{Q} \rightarrow \Sigma_{<}^{B}
\end{aligned}
$$

where $\epsilon_{l}: \operatorname{Symb}(\operatorname{Rec}(l)) \rightarrow Q$ is the counit function from Lemma 38. Above morphisms are well defined because, by Lemma $35, P=\operatorname{Symb}(\operatorname{Dep}(\Sigma))$, thus $l: Q \rightarrow \operatorname{Symb}(\operatorname{Dep}(\Sigma))$. Note that, in general, it may happen that $Q \neq$ $\operatorname{Symb}\left(\Sigma^{Q}\right)$ when $l$ is not injective on sort symbols.

Now, let $\Sigma_{<}^{T}$ be an algebraic signature with dependent symbols and $\theta_{A}: \Sigma_{<}^{T} \rightarrow$ $\Sigma_{<}^{A}$ and $\theta_{B}: \Sigma_{<}^{T} \rightarrow \Sigma_{<}^{B}$ be two AlgSigDep-morphisms. Let $T_{<}=\operatorname{DepSymb}\left(\Sigma_{<}^{T}\right)$. Like in the proof of Lemma 18 we define the monotone function $\theta: T \rightarrow P$ as $\theta(t)=\left\langle\operatorname{DepSymb}\left(\theta_{A}\right)(t), \operatorname{DepSymb}\left(\theta_{B}\right)(t)\right\rangle$ and the $\operatorname{Soset}_{\mathbf{w} f} \downarrow-$-morphism $u: T_{<} \rightarrow Q_{<}$as $u(t)=\left\langle\left[T_{<}, P_{<}, \theta\right]_{/ K}{ }^{[t]_{K} \downarrow}, \theta(t)\right\rangle$ for any $t \in T$. Mimicking the proof of Lemma 18, we learn that $u(t) \in Q$, the morphism $u$ is indeed a Soset $_{\mathbf{w}} \downarrow-$-morphism and that it is unique such, that $u ; \rho_{A}=\operatorname{Dep\operatorname {Symb}}\left(\theta_{A}\right)$ and $u ; \rho_{B}=\operatorname{DepSymb}\left(\theta_{B}\right)$. By Lemma 38, there exists the unique signature morphism $u^{\#}: \Sigma^{T} \rightarrow \Sigma^{Q}$ such that $\operatorname{Symb}\left(u^{\#}\right) ; \epsilon_{l}=u$. Using the above, since Symb is a functor and by Corollary 39 , we prove

$$
\begin{aligned}
\operatorname{Symb}\left(u^{\#} ; \rho_{A}^{\Sigma}\right) & =\operatorname{Symb}\left(u^{\#}\right) ; \operatorname{Symb}\left(\rho_{A}^{\Sigma}\right) \\
& =\operatorname{Symb}\left(u^{\#}\right) ; \operatorname{Symb}\left(\operatorname{Rec}_{\operatorname{Dep}(\Sigma)}(l) ; \pi_{A}^{\Sigma}\right) \\
& =\operatorname{Symb}\left(u^{\#}\right) ; \operatorname{Symb}\left(\operatorname{Rec}_{\operatorname{Dep}(\Sigma)}(l)\right) ; \operatorname{Symb}\left(\pi_{A}^{\Sigma}\right) \\
& =\operatorname{Symb}\left(u^{\#}\right) ; \epsilon_{l} ; l ; \pi_{A} \\
& =\operatorname{Symb}\left(u^{\#}\right) ; \epsilon_{l} ; \rho_{A} \\
& =u ; \rho_{A} \\
& =\operatorname{Symb}\left(\theta_{A}\right)
\end{aligned}
$$

Therefore, since the functor Symb is faithful (cf. Lemma 33), we get $u^{\#} ; \rho_{A}^{\Sigma}=$ $\theta_{A}$. Similarly we get $u^{\#} ; \rho_{B}^{\Sigma}=\theta_{B}$.

The colimits are inherited from AlgSig.

Theorem 43 The category AlgSigDep has all colimits. 
At this point we proved all that was needed to say that the category AlgSigDep is the natural extension of the category of algebraic signatures AlgSig that simply adds dependencies on signatures' symbols. The only cost we pay while shifting from one category to another is the loss of the final object. The other vital properties are preserved. Moreover, the limitation of dependency structure to well-founded strict orders seems to be reasonable and reflects the intuition one may have regarding the matter described in Sect. 2.

Here, this is also the right place to mention, that we imagine models of new signatures being exactly the same as these of the original ones. The whole "dependency thing" is about syntactical analysis, disregarded in models.

\section{Conclusion}

In the paper we proposed the category of algebraic signatures with symbol dependencies AlgSigDep. The proposal was proceeded by the long analysis of several possible orderings and proofs of the existence of the (co)limits in the respective categories. Then we proved the existence of all pushouts and pullbacks in AlgSigDep. Unfortunately the category lacks the final object. On the way we defined a product candidate in the category R-sets and p-morphisms, aka the category of transitive Kripke frames.

In the paper we used AlgSig and added the dependency structure. However, the construction is more generic than that and should work with most signature categories in the similar way.

Future work concerns the use of algebraic signatures enriched by dependency structure in covariant definition of signatures of generic modules in the architectural specifications framework.

Acknowledgment The author would like to thank Andrzej Tarlecki for his invaluable help and support.

\section{References}

[BST99] Michel Bidoit, Don Sannella, and Andrzej Tarlecki. Architectural specifications in CASL. In Proc. 7th Int. Conf. Algebraic Methodology and Software Technology (AMAST'98), Amazonia, Brazil, Jan. 1999, volume 1548 of LNCS, pages 341-357. Springer, 1999.

[Mar10] Grzegorz Marczyński. Algebraic signatures enriched by dependency structure (full version). Technical report, University of Warsaw, 2010. URL: http://www.mimuw .edu.pl/ gmarc/papers/wadt10.pdf.

[Seg70] K. Segerberg. Modal logics with linear alternative relations. Theoria, 36:301322, 1970.

[ST88] D. Sannella and A. Tarlecki. Toward formal development of programs from algebraic specifications: Implementations revisited. Acta Informatica, 25(3):233-281, 1988.

[ST97] Donald Sannella and Andrzej Tarlecki. Essential Concepts of Algebraic Specification and Program Development. Formal Asp. Comput., 9(3), 1997. 


\section{A Proofs of Lemmas and Theorems}

Here we give proofs of Lemmas and Theorems for whom there was no place in the main sections. Before we get to the proofs let us introduce a bit more of technicalities.

Definition 44 (Quotient of R-mset wrt. $\equiv$ ) Given an $R$-mset $\left[A_{R}, P_{R}, \mu\right]$ and an equivalence relation $\equiv \subseteq A^{2}$, a quotient $R$-mset is defined as an $R$-mset:

$$
\left[A_{R}, P_{R}, \mu\right]_{\equiv \equiv}=\left[A_{R}^{\prime}, P_{R}, \mu^{\prime}\right]
$$

where $A^{\prime}=A_{/ \equiv}, R_{A^{\prime}}$ is defined by

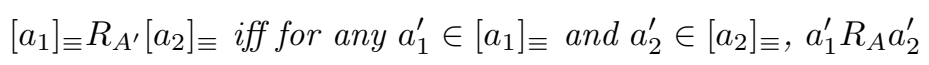

and the labeling

$$
\mu^{\prime}\left([a]_{\equiv}\right)=\mu(a)
$$

where $[a]_{\equiv}$ is the equivalence class of a wrt. $\equiv$.

Definition 45 (Kernel of an R-mset) Given an R-mset $\left[A_{R}, P_{R}, \mu\right]$, its kernel is defined as

$$
\left[A_{R}, P_{R}, \mu\right]_{/ K}=\left[A_{R}, P_{R}, \mu\right]_{/ K\left(\left[A_{R}, P_{R}, \mu\right]\right)}
$$

Elements of the kernel are equivalence classes, for $a \in A,[a]_{K}=[a]_{K\left(\left[A_{R}, P_{R}, \mu\right]\right)}$.

Lemma 46 Given an $R$-mset $\left[A_{R}, P_{R}, \mu\right]$

$$
K\left(\left[A_{R}, P_{R}, \mu\right]_{/ K}\right)=i d_{A_{/ K\left(\left[A_{R}, P_{R}, \mu\right]\right)}}
$$

Proof: Let $\sim^{\prime} \subseteq\left(A_{/ K\left(\left[A_{R}, P_{R}, \mu\right]\right.}\right)^{2}$ be a dependency bisimulation on $\left[A_{R}, P_{R}, \mu\right]_{/ K}$, then $\sim \subseteq A^{2}$, defined as $a_{1} \sim a_{2}$ iff $\left[a_{1}\right]_{K} \sim^{\prime}\left[a_{2}\right]_{K}$, for $a_{1}, a_{2} \in A$, is a dependency bisimulation on $\left[A_{R}, P_{R}, \mu\right]$. Therefore, $\sim \subseteq K\left(\left[A_{R}, P_{R}, \mu\right]\right)$ and for any $a_{1}, a_{2} \in A$, if $\left[a_{1}\right]_{K} \sim^{\prime}\left[a_{2}\right]_{K}$ then $a_{1} \sim a_{2}$ and $\left\langle a_{1}, a_{2}\right\rangle \in K\left(\left[A_{R}, P_{R}, \mu\right]\right)$, thus $\left[a_{1}\right]_{K}=\left[a_{2}\right]_{K}$ and $\sim^{\prime}=i d_{A_{/ K\left(\left[A_{R}, P_{R}, \mu\right]\right)}}$.

Lemma 47 Given an $R$-mset $\left[A_{R}, P_{R}, \mu\right]$ and $a \in A$,

$$
K\left(\left[A_{R}, P_{R}, \mu\right]^{a} \downarrow\right)=K\left(\left[A_{R}, P_{R}, \mu\right]\right) \cap\left(\left[A_{R}, P_{R}, \mu\right]^{a} \downarrow\right)^{2}
$$

Proof: The proof is straightforward. Let us just notice that the kernel relation is based solely on the structure down from the given element wrt. $R$ in the R-mset.

Corollary 48 Given an $R$-mset $\left[A_{R}, P_{R}, \mu\right]$, and $a \in A$, it holds that

$$
\left(\left[A_{R}, P_{R}, \mu\right]^{a} \downarrow\right)_{/ K}=\left(\left[A_{R}, P_{R}, \mu\right]_{/ K}\right)^{[a]_{K}} \downarrow
$$


Lemma 49 Given an $\mathbf{R s e t} \downarrow$-morphism $f: A_{R} \rightarrow B_{R}$, and an element $a \in A$, the reduct $\left.f\right|_{A_{R}{ }^{a} \downarrow}: A_{R}{ }^{a} \downarrow \rightarrow B_{R}$ is also an $\mathbf{R s e t} \downarrow$-morphism.

Proof: The reduct $\left.f\right|_{A_{R}{ }^{a} \downarrow}$ is monotone, because $f$ is so. Since $R$ is transitive, for any $a^{\prime} \in A_{R}{ }^{a} \downarrow$, all $a^{\prime \prime} R_{A} a^{\prime}$ are in $A_{R}{ }^{a} \downarrow$ as well and so $\left.f\right|_{A_{R}{ }^{a} \downarrow}$ meets the requirement (2) of Def. 2 , as $f$ does.

Lemma 50 (Rset $\downarrow$-morphisms Preserve Dependency Bisimulations) Consider two $R$-msets $\left[A_{R}, P_{R}, \mu\right]$ and $\left[A_{R}^{\prime}, P_{R}, \mu^{\prime}\right]$ and a Rset $\downarrow$-morphism $f: A_{R} \rightarrow A_{R}^{\prime}$, such that $\mu=f ; \mu^{\prime}$. If $\sim \subseteq A^{2}$ is a dependency bisimulation on $\left[A_{R}, P_{R}, \mu\right]$, then $f(\sim) \subseteq f(A)^{2}$ is a dependency bisimulation on $\left[f(A)_{R}, P_{R},\left.\mu\right|_{f(A)}\right]$, where $R_{f(A)}=R_{\left.A^{\prime}\right|_{f(A)}}$.

Proof: Let $\sim^{\prime}=f(\sim)$. Then, given $a_{1}, a_{2} \in A, a_{1} \sim a_{2}$, we have $f\left(a_{1}\right) \sim^{\prime} f\left(a_{2}\right)$. By $\mu=f ; \mu^{\prime}$, we have $\mu^{\prime}\left(f\left(a_{1}\right)\right)=\mu^{\prime}\left(f\left(a_{2}\right)\right)$ and for any $p^{\prime} \in P, p^{\prime} R \mu\left(f\left(a_{1}\right)\right)$ and $b_{1}^{\prime} \in A^{\prime}$, if $\mu^{\prime}\left(b_{1}^{\prime}\right)=p^{\prime}$ and $b_{1}^{\prime} R f\left(a_{1}\right)$, by requirement (2) of Def. 2 , there exists $a_{1}^{\prime} \in A, a_{1}^{\prime} R a_{1}, f\left(a_{1}^{\prime}\right)=b_{1}$ and, since $\sim$ is a dependency bisimulation, there exists $a_{2}^{\prime} R a_{2}, a_{1}^{\prime} \sim a_{2}^{\prime}$, thus $f\left(a_{2}^{\prime}\right) R f\left(a_{2}\right)$ and $f\left(a_{2}^{\prime}\right) \sim^{\prime} f\left(a_{1}^{\prime}\right)=b_{1}^{\prime}$, hence $b_{1}^{\prime} \in f(A)$. By symmetry, this proves that $f(\sim)$ is a dependency bisimulation on $\left[f(A)_{R}, P_{R}, \mu^{\prime}\right]$.

Corollary 51 Consider two R-msets $\left[A_{R}, P_{R}, \mu\right]$ and $\left[A_{R}^{\prime}, P_{R}, \mu^{\prime}\right]$ and a surjective Rset $\downarrow$-morphism $f: A_{R} \rightarrow A_{R}^{\prime}$, such that $\mu=f ; \mu^{\prime}$. If $\sim \subseteq A^{2}$ is a dependency bisimulation on $\left[A_{R}, P_{R}, \mu\right]$, then $f(\sim) \subseteq A^{\prime 2}$ is a dependency bisimulation on $\left[A_{R}^{\prime}, P_{R}, \mu^{\prime}\right]$.

Lemma 52 Given two $R$-msets $\left[A_{R}, P_{R}, \mu\right]$ and $\left[A_{R}^{\prime}, P_{R}, \mu^{\prime}\right]$ and a Rset $\downarrow$-morphism $f: A_{R} \rightarrow A_{R}^{\prime}$, such that $\mu=f ; \mu^{\prime}$, it holds that

$$
K(f) \subseteq K\left(\left[A_{R}, P_{R}, \mu\right]\right)
$$

where $K(f) \subseteq A^{2}$ is the kernel relation for the function $f$. Moreover, if $K\left(\left[A_{R}^{\prime}, P_{R}, \mu\right]\right)=$ $i d_{A^{\prime}}$, then

$$
K(f)=K\left(\left[A_{R}, P_{R}, \mu\right]\right)
$$

Proof: A Rset $\downarrow$-morphism $f$ weakly reflects R-set down-closures. Therefore, if $a_{1}, a_{2} \in A$ are such that $f\left(a_{1}\right)=f\left(a_{2}\right)$, then $\mu\left(a_{1}\right)=\mu\left(a_{2}\right)$ and for any $p^{\prime} R \mu\left(a_{1}\right)$ and any $a_{3} R a_{1}$ such that $\mu\left(a_{3}\right)=p^{\prime}$, since $f\left(a_{3}\right) R f\left(a_{1}\right)=f\left(a_{2}\right)$ there exists $a_{4} R a_{2}, f\left(a_{4}\right)=f\left(a_{3}\right)$. By symmetry, this implies that $K(f)$ is a dependency bisimulation on $\left[A_{R}, P_{R}, \mu\right]$ and since $K\left(\left[A_{R}, P_{R}, \mu\right]\right)$ is the greatest such relation, $K(f) \subseteq K\left(\left[A_{R}, P_{R}, \mu\right]\right)$.

When additionally $K\left(\left[A_{R}^{\prime}, P_{R}, \mu\right]\right)=i d_{A^{\prime}}$, let $\sim \subseteq A^{2}$ be a dependency bisimulation on $\left[A_{R}, P_{R}, \mu\right]$ and $a_{1}, a_{2} \in A$. If $a_{1} \sim a_{2}$ then, by definition of $f$, $f\left(a_{1}\right) f(\sim) f\left(a_{2}\right)$ and, since $f(\sim)$ is a dependency bisimulation on $\left[f(A)_{R}, P_{R},\left.\mu\right|_{f(A)}\right]$ (cf. Lemma 50), $f(\sim) \subseteq K\left(\left[f(A)_{R}, P_{R}, \mu_{f(A)}\right]\right) \subseteq K\left(\left[A_{R}^{\prime}, P_{R}, \mu^{\prime}\right]\right)=i d_{A^{\prime}}$, thus 
$f\left(a_{1}\right)=f\left(a_{2}\right)$, therefore $K\left(\left[A_{R}, P_{R}, \mu\right]\right) \subseteq K(f)$. Together with the previous result this gives $K(f)=K\left(\left[A_{R}, P_{R}, \mu\right]\right)$.

\section{Proof of Theorem 13:}

The relations are reflexive, therefore there exist unique morphisms from any of their objects to the singleton ordered by identity.

Proof of Theorem 14: Since the relations in objects of Soset $\downarrow$ (and Soset $_{w f} \downarrow$ ) are irreflexive, their morphisms must not glue together any elements being in relation. Hence, if the final object existed, there would be an injective map from any ordinal (represented as an R-set with natural strict well-founded "ordering") into it. Hence, such a final object can not be a proper set.

Proof of Theorem 16: Given two morphisms $f, g: A_{R} \rightarrow B_{R}$ in Rset $\downarrow$, their equalizer is an inclusion $e: C_{R} \rightarrow A_{R}$, where

$$
C=\left\{a \in A \mid \text { for all } a^{\prime} \in A_{R}{ }^{a} \downarrow, f\left(a^{\prime}\right)=g\left(a^{\prime}\right)\right\}
$$

See Def. 3 for the definition of closed down sub R-set $A_{R}{ }^{a} \downarrow$ induced by $a$. The relation is defined as $R_{C}=\left.R_{A}\right|_{C}$. Trivially, $e$ is an Rset $\downarrow$-morphism. Let us check that it is universal. Let $h: D_{R} \rightarrow A_{R}$ be such Rset $\downarrow$-morphism that $h ; f=h ; g$. We need to find the unique $u: D_{R} \rightarrow C_{R}$, such that $u ; e=h$. Since $e$ is an inclusion and because $h(D) \subseteq C$, putting $u(d)=h(d)$ for $d \in D$ yields the only such morphism. The inclusion $h(D) \subseteq C$ is the consequence of the fact that $h$, as an Rset $\downarrow$-morphism, weakly reflects R-set down-closures. To complete the proof, it is enough to notice that if $A_{R}$ and $B_{R}$ are in Preord $\downarrow$, Soset $\downarrow$ or $\operatorname{Soset}_{\mathbf{w f}} \downarrow$, respectively, then so is $C_{R}$.

Lemma 53 Every $R$-mset component of $\left\langle\left[X_{R}, P_{R}, \mu\right], p\right\rangle \in\left(A \prod B\right)_{R}$ has a distinguished top-element $x$, i.e. there exists exactly one $x \in X$, such that $\mu(x)=p$ and for all $x^{\prime} \neq x \in X, x^{\prime} R x$.

Proof: By contradiction, let $x_{1}$ be another such element, i.e. $x_{1} \neq x$ and for all $x^{\prime} \neq x_{1} \in X, x^{\prime} R x_{1}$. As a consequence $x R x_{1}$ and $x_{1} R x$, thus, since $R$ is transitive, $\left\langle x, x_{1}\right\rangle \in K\left(\left[X_{R}, P_{R}, \mu\right]\right)=i d_{X}$. Contradiction.

Lemma 54 (Product Candidate is Self-adequate) The class $A \prod B$ is selfadequate, meaning that for all $\left\langle\left[X_{R}, P_{R}, \mu\right], p\right\rangle \in A \prod B$ and for all $x \in X$

$$
\left\langle\left[X_{R}, P_{R}, \mu\right]^{x} \downarrow, \mu(x)\right\rangle \in A \prod B
$$

Proof of Lemma 18: Let us assume that $A \prod B$ is a set. For the notation convenience we name

$$
\left\langle Q, R_{Q}\right\rangle=\left\langle A \prod B, R_{A} \prod_{B}\right\rangle
$$


We notice that, by definition, $R_{Q}$ is transitive, which makes $Q_{R}$ indeed an Rset $\downarrow$-object. Moreover, both $\rho_{A}$ and $\rho_{B}$ are Rset $\downarrow$ morphisms. They obviously preserve the relation. Let $q_{1}, q_{2} \in Q$ be $q_{1}=\left\langle\left[X_{R}^{1}, P_{R}, \mu_{1}\right], p_{1}\right\rangle$ and $q_{2}=$ $\left\langle\left[X_{R}^{2}, P_{R}, \mu_{2}\right], p_{2}\right\rangle$, such that $q_{1} R_{Q} q_{2}$, then, by definition, $p_{1} R_{P} p_{2}$, thus $\left(\rho_{A}\left(q_{1}\right)\right) R_{A}\left(\rho_{A}\left(q_{2}\right)\right)$ (and similarly for $\rho_{B}$ ). They also meet the requirement (2) of Def. 2, which makes them Rset $\downarrow$-morphisms. Namely, given $q=\left\langle\left[X_{R}, P_{R}, \mu\right], p\right\rangle \in Q$ and $a^{\prime} \in A$, $a^{\prime} R_{A} \rho_{A}(q)$, by definition of $Q$ there exists $x \in X, \mu(x)=p, \mu ; \pi_{A}(x)=a$ and, since $\mu ; \pi_{A}$ is a Rset $\downarrow$-morphism, there exists $x^{\prime} R x, \mu ; \pi_{A}\left(x^{\prime}\right)=a^{\prime}$. By self-adequacy of $Q$ (cf. Lemma 54), there is $q^{\prime}=\left\langle\left[X_{R}, P_{R}, \mu\right]^{x^{\prime}} \downarrow, \mu\left(x^{\prime}\right)\right\rangle$, and of course $q^{\prime} R q$ and $\rho_{A}\left(q^{\prime}\right)=\pi_{A}\left(\mu\left(x^{\prime}\right)\right)=a^{\prime}$. For $\rho_{B}$ the proof goes likewise.

Now we show that for each object $T_{R} \in$ Rset $\downarrow$ and two morphisms $\theta_{A}: T_{R} \rightarrow$ $A_{R}$ and $\theta_{B}: T_{R} \rightarrow B_{R}$ in Rset $\downarrow$, there exists a unique Rset $\downarrow$-morphism $u: T_{R} \rightarrow$ $Q_{R}$ such that $u ; \rho_{A}=\theta_{A}$ and $u ; \rho_{B}=\theta_{B}$.

Let an Rset $\downarrow$-object $T_{R}$ and morphisms $\theta_{A}$ and $\theta_{B}$ be as described above. We define a function $\theta: T \rightarrow P$ as

$$
\theta(t)=\left\langle\theta_{A}(t), \theta_{B}(t)\right\rangle
$$

The monotonicity of $\theta$ follows the monotonicity of $\theta_{A}$ and $\theta_{B}$ and the definition of $R_{P}$. By definition, it holds that $\theta ; \pi_{A}=\theta_{A}$ and $\theta ; \pi_{B}=\theta_{B}$. We notice that $\left[T_{R}, P_{R}, \theta\right]$ is an R-mset. Let

$$
\left[T_{R}^{\prime}, P_{R}, \theta^{\prime}\right]=\left[T_{R}, P_{R}, \theta\right]_{/ K}
$$

It is easy to prove that $\theta^{\prime} ; \pi_{A}$ and $\theta^{\prime} ; \pi_{B}$ are $\mathbf{R s e t} \downarrow$-morphisms. Let the morphism $u: T_{R} \rightarrow Q_{R}$ be defined as

$$
u(t)=\left\langle\left[T_{R}, P_{R}, \theta\right]_{/ K}{ }^{[t]_{K}} \downarrow, \theta(t)\right\rangle
$$

for any $t \in T$, where $[t]_{K}$ is an equivalence class of $t$ wrt. $K\left(\left[T_{R}, P_{R}, \theta\right]\right)$ (cf. Def. 12 and Def. 45). Before we proceed with the proof let us simplify the notation by naming

$$
\left[T_{R}^{t}, P_{R}, \theta_{t}\right]=\left[T_{R}, P_{R}, \theta\right]_{/ K}[t]_{K} \downarrow
$$

for $t \in T$.

Let us show that for every $t \in T, u(t) \in Q$. The R-mset $\left[T_{R}^{t}, P_{R}, \theta_{t}\right]$ indeed meets all requirements from the definition of $Q$. By Def. 10, $\theta_{t}=\theta_{T^{t}}$ and, since $\theta^{\prime} ; \pi_{A}$ and $\theta^{\prime} ; \pi_{B}$ are Rset $\downarrow$-morphisms, by Lemma $49 \theta_{t} ; \pi_{A}$ and $\theta_{t} ; \pi_{B}$ are also Rset $\downarrow$-morphisms. Moreover, the element $[t]_{K}$ is such that for all $x \in T^{t}$, (x) $R\left([t]_{K}\right)$ and by Lemma 46 and Corollary $48, K\left(\left[T_{R}^{t}, P_{R}, \theta_{t}\right]\right)=i d_{\left[T_{R}^{t}, P_{R}, \theta_{t}\right]}$. Now, let us check that $u$ is monotone. Let $t^{\prime}, t \in T$ and $t^{\prime} R_{T} t$. To prove that $u\left(t^{\prime}\right) R_{Q} u(t)$ we need to show that there exists $x^{\prime} \in T^{t}, \theta_{t}\left(x^{\prime}\right)=\theta\left(t^{\prime}\right)$ and that $\left[T_{R}^{t^{\prime}}, P_{R}, \theta_{t^{\prime}}\right]=\left[T_{R}^{t}, P_{R}, \theta_{t}\right]^{x^{\prime} \downarrow}$. Let us take $x^{\prime}=\left[t^{\prime}\right]_{K}$. Obviously $\theta_{t}\left(\left[t^{\prime}\right]_{K}\right)=$ $\theta\left(t^{\prime}\right)$. The second requirement, $\left[T_{R}^{t^{\prime}}, P_{R}, \theta_{t^{\prime}}\right]=\left[T_{R}^{t}, P_{R}, \theta_{t}\right]^{\left[t^{\prime}\right]_{K} \downarrow}$ also holds because $\left(\left[t^{\prime}\right]_{K}\right) R_{T^{t}}\left([t]_{K}\right)$ and by Lemma 11. It is trivial to show that $u ; \rho_{A}=\theta_{A}$ and $u ; \rho_{B}=\theta_{B}$. To finish the proof we need to show that a function $u$ is a Rset $\downarrow-$ morphism and that the choice of $u$ is unique. 
We prove that the function $u$ is a $\mathbf{R s e t} \downarrow$-morphism. We already have shown that it is monotone. The requirement (2) of Def. 2 says that for any $t \in T$ and $q^{\prime}=\left\langle\left[X_{R}^{\prime}, P_{R}, \mu^{\prime}\right], p^{\prime}\right\rangle \in Q$ such that $\left(q^{\prime}\right) R_{Q}(u(t))$, there must exist $t^{\prime} \in T, t^{\prime} R t$ and $u\left(t^{\prime}\right)=q^{\prime}$. Since $\left(q^{\prime}\right) R_{Q}(u(t))$, there exists $x^{\prime} \in T^{t}$ that $\theta_{t}\left(x^{\prime}\right)=p^{\prime}$ and, by definition of $u$, there exists $t^{\prime} \in T$ that $\left[t^{\prime}\right]_{K}=x^{\prime}$ and $t^{\prime} R t$. Of course $\theta\left(t^{\prime}\right)=p^{\prime}$. Moreover, by definition of $R_{Q},\left[X_{R}^{\prime}, P_{R}, \mu^{\prime}\right]=\left[T_{R}^{t}, P_{R}, \theta_{t}\right]^{\left[t^{\prime}\right]_{K}} \downarrow$ and by Lemma 11 we have $\left[T_{R}^{t}, P_{R}, \theta_{t}\right]^{\left[t^{\prime}\right]_{k}} \downarrow=\left[T_{R}^{t^{\prime}}, P_{R}, \theta_{t^{\prime}}\right]$. This proves that $q^{\prime}=u\left(t^{\prime}\right)$.

To show the uniqueness of $u$ such that $u ; \theta_{A}=\rho_{A}$ and $u ; \theta_{B}=\rho_{B}$ let us have some Rset $\downarrow$-morphism $u^{\prime}: T_{R} \rightarrow Q_{R}$ that $u ; \theta_{A}=\rho_{A}$ and $u ; \theta_{B}=\rho_{B}$. For any $t \in T, u^{\prime}(t)=\left\langle\left[X_{R}, P_{R}, \mu\right], \theta(t)\right\rangle$, for some R-set $X_{R}$ and a monotone function $\mu: X_{R} \rightarrow P_{R}$. Let us define a surjective Rset $\downarrow$ morphism $u_{t}^{\prime}: T_{R}{ }^{t} \downarrow \rightarrow X_{R}$ as follows. For any $t^{\prime} \in T_{R}{ }^{t} \downarrow$ we have $\left(u^{\prime}\left(t^{\prime}\right)\right) R_{Q}(u(t))$, thus $u^{\prime}\left(t^{\prime}\right)=\left\langle\left[X, P_{R}, \mu\right]^{x^{\prime}} \downarrow, \theta\left(t^{\prime}\right)\right\rangle$ for exactly one $x^{\prime} \in X$ (cf. Lemma 53), let

$$
u_{t}^{\prime}\left(t^{\prime}\right)=x^{\prime}
$$

The morphism $u^{\prime}$ meets both requirements of Def. 2 - it is monotone and, since $Q$ is self adequate (cf. Lemma 54), for each $x^{\prime \prime} \in X$, if $x^{\prime \prime} R_{X} x^{\prime}$ then there exists $q^{\prime \prime} \in Q$ such that $\left(q^{\prime \prime}\right) R_{Q}\left(u^{\prime}\left(t^{\prime}\right)\right)$ and, since $u^{\prime}$ is a Rset $\downarrow$-morphism, there must exist $t^{\prime \prime} \in T$ such that $t^{\prime \prime} R t^{\prime}$ and $u^{\prime}\left(t^{\prime \prime}\right)=q^{\prime \prime}=\left\langle\left[X_{R}, P_{R}, \mu\right]^{x^{\prime \prime}} \downarrow, \theta\left(t^{\prime \prime}\right)\right\rangle$, thus $u_{t}^{\prime}\left(t^{\prime \prime}\right)=x^{\prime \prime}$. This also proves that $u_{t}^{\prime}$ is surjective. Hence, there is a bijection between $X$ and $\left(T_{R}^{t} \downarrow\right) / K\left(u_{t}^{\prime}\right)$, where $K\left(u_{t}^{\prime}\right)$ is the kernel of the function $u_{t}^{\prime}$. By Lemma 52 , since $u_{t}^{\prime}$ is surjective and $K\left(\left[X_{R}, P_{R}, \mu\right]\right)=i d_{X}$,

$$
K\left(u_{t}^{\prime}\right)=K\left(\left[T_{R}{ }^{t} \downarrow, P_{R}, \theta_{T_{R} \downarrow} \downarrow\right]\right)=K\left(\left[T_{R}, P_{R}, \theta\right]^{t} \downarrow\right)
$$

This means that $\left[X, P_{R}, \mu\right]=\left(\left[T_{R}, P_{R}, \theta\right]^{t} \downarrow\right) / K$. By Corollary 48 , we get $\left[X, P_{R}, \mu\right]=$

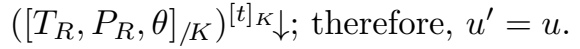

This shows the uniqueness of $u$ and completes the proof that $\left\langle Q, R_{Q}\right\rangle$, together with projections $\rho_{A}$ and $\rho_{B}$, is the product of $A_{R}$ and $B_{R}$ in Rset $\downarrow$.

Proof of Lemma 19: (reflexive) If $R_{A}$ and $R_{B}$ are reflexive, then clearly $R_{P}$ is also reflexive. Let $q=\left\langle\left[X_{R}, P_{R}, \mu\right], p\right\rangle \in A \prod B$. By Lemma 53 there exists a top-element $x \in X$ and we notice that $\left[X_{R}, P_{R}, \mu\right]=\left[X_{R}, P_{R}, \mu\right]^{x} \downarrow$. By reflexivity of $R_{P}$ we have $p R p$. All together this makes $q R q$ (cf. Def.17).

(irreflexive) Let $R_{A}$ and $R_{B}$ be irreflexive. By contradiction. Let $q=\left\langle\left[X_{R}, P_{R}, \mu\right], p\right\rangle \in$ $A \prod B$ and $q R q$. Thus, $p R p$; therefore, $\left(\pi_{A}(p) R_{A}\left(\pi_{A}(p)\right)\right.$, which contradicts the irreflexivity of $R_{A}$.

(asymmetric) Let $R_{A}$ and $R_{B}$, thus also $R_{P}$, be asymmetric. Let $q=\left\langle\left[X_{R}, P_{R}, \mu\right], p\right\rangle, q^{\prime}=$ $\left\langle\left[X_{R}^{\prime}, P_{R}, \mu^{\prime}\right], p^{\prime}\right\rangle \in A \prod B$, and let $q R q^{\prime}$. This means that $p R p^{\prime}$ and, by asymmetry, it doesn't hold that $p^{\prime} R p$. Therefore, by definition of $R_{A \prod B}$ (cf. Def. 53), it doesn't hold that $q^{\prime} R q$.

(strict well-founded) Let $R_{A}$ and $R_{B}$ be strict well-founded relations. In this case $R_{P}$ is also strict well founded. Let $C \subseteq A \prod B$ be a descending chain wrt. $R_{A \prod B}$. If it is empty, then it is finite. Otherwise, let $q=\left\langle\left[X_{R}, P_{R}, \mu\right], p\right\rangle \in C$. 
Let us notice that due to Def. 17 , for any $q^{\prime} \in C, q^{\prime} R q$, there exists $x^{\prime} \in X$ such that $q^{\prime}=\left\langle\left[X_{R}, P_{R}, \mu\right]^{x^{\prime}} \downarrow, \mu\left(x^{\prime}\right)\right\rangle$. It means that if $C^{q} \downarrow$ was infinite, then $X_{R}{ }^{x^{\prime}} \downarrow$ would also be so. However, this is not the case, because $\mu\left(X_{R}\right)$ is finite (as a chain wrt. well-founded $R_{P}$ ) and $\mu$ is monotone. Therefore, $C^{q} \downarrow$ is finite and $R_{A \prod B}$ is strict well-founded.

Proof of Theorem 20: By Lemma 19 we know that $<_{A} \prod_{B}$ is a well-founded strict order, thus in fact $A \prod B$, if proved to be a set, is the Soset $_{\mathbf{w} f} \downarrow \downarrow$-object. To show that it is indeed a product we need to show that $A \prod B$ is a set to be able to use Lemma 18. To do so, it is enough to bound the number of $A \prod B$ elements that share the given label $p \in P$ (using notation from Def. 17). Given $q=\left\langle\left[X_{<}, P_{<}, \mu\right], p\right\rangle \in A \prod B$ and a label $p^{\prime} \in P_{<}{ }^{p} \downarrow$, let us bound the cardinality of $\mu^{-1}\left(p^{\prime}\right)$ by cases:

- (base case) if $P_{<} p^{\prime}=\{p\}$, then $p^{\prime}=p$ and $\left|\mu^{-1}\left(p^{\prime}\right)\right|<=1$, i.e. $l_{p^{\prime}}=1$, because there may be only one element labeled by $p^{\prime}$ distinct wrt. the kernel relation (cf. Def. 12);

- (induction step) otherwise, let $\mathcal{L}=\sum_{p^{\prime \prime}<p^{\prime}} l_{p^{\prime \prime}}$, where $l_{p^{\prime \prime}}$ is the bound of $\mu^{-1}\left(p^{\prime \prime}\right)$ for $p^{\prime \prime}<p^{\prime}$, then $\left|\mu^{-1}\left(p^{\prime}\right)\right|<=2^{\mathcal{L}}$, i.e. $l_{p^{\prime}}=2^{\mathcal{L}}$; it is impossible to have more elements labeled by $p^{\prime}$ distinct wrt. the kernel relation, than all combinations of elements lower wrt. $<_{X}$.

The cardinal number $l_{p^{\prime}}$ is well defined for every $p^{\prime} \in P_{<}{ }^{p} \downarrow$, because $<_{X}$ is a well-founded strict order. Finally, by definition of $<_{A} \prod_{B}$, we conclude that the cardinality of the set of elements that share the label $p$ is bounded by $l_{p}$, therefore, $A \prod B$ is a set. By Lemma 18 a Soset $_{\mathbf{w} f} \downarrow$-object $\left\langle A \prod B,<_{A} \prod_{B}\right\rangle$ together with morphisms $\rho_{A}$ and $\rho_{B}$, as defined in Def. 17, is a product of $A_{<}$, $B_{<}$in Soset $_{\mathbf{w f}} \downarrow$.

Lemma 55 Given two $\mathbf{R s e t}_{\downarrow}$-morphisms $f, g: A_{R} \rightarrow B_{R}$ and a relation $\sim \subseteq$ $B^{2}$ defined as $b_{1} \sim b_{2}$ iff there exists $a \in A$ such that $b_{1}=f(a)$ and $b_{2}=g(a)$ and its reflexive, symmetric and transitive closure $\equiv=\operatorname{Trans}(\operatorname{Sym}(\operatorname{Ref}(\sim)))$, it holds that: for any $b_{1}, b_{2} \in B$, if $b_{1} R_{B} b_{2}$ then for any $b_{2}^{\prime} \equiv b_{2}$ there exists $b_{1}^{\prime} \equiv b_{1}$ such that $b_{1}^{\prime} R_{B} b_{2}^{\prime}$.

Proof: For any $b_{2} \equiv b_{2}^{\prime}$ there exists a path from $b_{2}$ to $b_{2}^{\prime}$ in the undirected graph $\operatorname{Graph}(f) \cup \operatorname{Graph}(g)$. Let $a_{2} \in A$ be such that $f\left(a_{2}\right)=b_{2}$ and let $b_{1} R_{B} b_{2}$. Since $f$ is Rset $\downarrow$-morphism, by requirement (2) of Def. 2, there exists $a_{1} \in A$ such that $f\left(a_{1}\right)=b_{1}$ and $a_{1} R_{A} a_{2}$. Since $g$ is also an Rset $\downarrow$-morphism, by requirement (1) of the same definition, it is monotone, i.e., $g\left(a_{1}\right) R_{A} g\left(a_{2}\right)$ and $g\left(a_{1}\right) \equiv b_{1}$ and $g\left(a_{2}\right) \equiv b_{2}$. The above procedure executed along the path between $b_{2}$ and $b_{2}^{\prime}$ (the same that served the transitive closure in definition of $\equiv$ ) results in existence of the required $b_{1}^{\prime} \equiv b_{1}$ such that $b_{1}^{\prime} R_{B} b_{2}^{\prime}$.

Proof of Lemma 22: Let $f, g: A_{R} \rightarrow B_{R}$ be two Rset $\downarrow$-morphisms and $e: B \rightarrow C$ be their coequalizer in Set. Let us notice that $C=B_{/ \equiv}$, where 
$\equiv$ is an equivalence defined as in Lemma 55 . We need to show that $R_{C}$ is transitive and that $e$ meets both conditions of Def. 2. In fact $R_{C}$ is transitive. Let $c_{1} R_{C} c_{2} R_{C} c_{3}$. Function $e$ as a coequalizer in Set is surjective, thus there exist $b_{1}, b_{2}, b_{2}^{\prime}, b_{3} \in B$ such that $e\left(b_{1}\right)=c_{1}, e\left(b_{2}\right)=e\left(b_{2}^{\prime}\right)=c_{2}, e\left(b_{3}\right)=c_{3}$ and $b_{1} r_{B} b_{2}$ and $b_{2}^{\prime} R_{B} b_{3}$. By Lemma 55 , since $b_{2} \equiv b_{2}^{\prime}$, there exists $b_{1}^{\prime} \equiv b_{1}$ such that $b_{1}^{\prime} R_{B} b_{2}^{\prime}$. Relation $R_{B}$ is transitive thus $b_{1}^{\prime} R_{B} b_{3}$. Of course, since $e$ is the coequalizer of $f$ and $g$, we get $e\left(b_{1}^{\prime}\right)=e\left(b_{1}\right)=c_{1}$. Therefore $c_{1} R_{C} c_{3}$. Function $e$ trivially meets the first condition of Def. 2, because $R_{C}=e\left(R_{B}\right)$. To prove the second condition let $b_{2} \in B$ and $c_{1} \in C$ such that $c_{1} R_{C} e\left(b_{2}\right)$. From the surjectivity of $e$ and the definition of $R_{C}$ we get the existence of $b_{2}^{\prime}, b_{1}^{\prime} \in B$ such that $e\left(b_{1}^{\prime}\right)=c_{1}, e\left(b_{2}^{\prime}\right)=e\left(b_{2}\right)$ and $b_{1}^{\prime} R_{B} b_{2}^{\prime}$. Therefore, $b_{2}^{\prime} \equiv b_{2}$ and by Lemma 55, there exists $b_{1} \in B$ such that $b_{1} \equiv b_{1}^{\prime}$ and $b_{1} R_{B} b_{2}$, and accordingly $e\left(b_{1}\right)=e\left(b_{1}^{\prime}\right)=c_{1}$.

Proof of Theorem 23: The coequalizer of two morphisms $f, g: A_{R} \rightarrow B_{R}$ in Rset $\downarrow$ is $e: B_{R} \rightarrow C_{R}$, where $e$ is the coequalizer of $f$ and $g$ in Set and a relation $R_{C}=e\left(R_{B}\right)$. The universal properties of $e$ in Rset $\downarrow$ are inherited from Set. Namely, given a morphism $h: B_{R} \rightarrow D_{R}$ in Rset $\downarrow$ such that $f ; h=g ; h$, there exists a unique function $k: C \rightarrow D$ such that $e ; k=h$. It is monotone. Given $c_{1}, c_{2} \in C, c_{1} R_{C} c_{2}$ since $e$ in surjective (as a coequalizer in Set) and monotone there exist $b_{1}, b_{2} \in B$ such that $b_{1} R_{B} b_{2}$ and $e\left(b_{1}\right)=c_{1}$ and $e\left(b_{2}\right)=c_{2}$. We have $h\left(b_{1}\right) R_{D} h\left(b_{2}\right)$, because $h$ is monotone, and finally

$$
k\left(c_{1}\right)=k\left(e\left(b_{1}\right)\right)=h\left(b_{1}\right) R_{D} h\left(b_{2}\right)=k\left(e\left(b_{1}\right)\right)=k\left(c_{2}\right)
$$

Function $k$ also meets the requirement (2) of Def. 2. Let $c_{1} \in C$ and $d_{2} \in D$ be such that $d_{2} R_{D} k\left(c_{1}\right)$. Since $h$ is a p-morphism, for any $b_{1} \in B$ such that $h\left(b_{1}\right)=k\left(c_{1}\right)$ there exits $b_{2} \in B, b_{2} R_{B} b_{1}$ and $h\left(b_{2}\right)=d_{2}$. Let us choose the $b_{1}$ such that $e\left(b_{1}\right)=c_{1}$. It exists because $e$ is surjective. Of course $h\left(b_{1}\right)=$ $k\left(e\left(b_{1}\right)\right)=k\left(c_{1}\right)$, so there is $b_{2}$ with properties given above. Function $e$ is monotone, thus, $e\left(b_{2}\right) R_{C} e\left(b_{1}\right)$. Moreover, $k\left(e\left(b_{2}\right)\right)=h\left(b_{2}\right)=d_{2}$. The two, above shown, properties of function $k$ make it an Rset $\downarrow$-morphism.

Due to Lemma 22, $e$ is an $\mathbf{R s e t} \downarrow$-morphism and $e\left(R_{B}\right)$ is a transitive relation. Note that reflexivity of $R_{B}$ guarantees reflexivity of $e\left(R_{B}\right)$.

Proof of Theorem 24: Let us show a counterexample. A strictly ordered set $\langle\mathbb{N}$, prev $\rangle$, where prev $\subseteq \mathbb{N} \times \mathbb{N}$ is defined as prev $=\{\langle n+1, n\rangle \mid n \in \mathbb{N}\}$, is a Soset $\downarrow$-object. Let $f, g:\langle\mathbb{N}$, prev $\rangle \rightarrow\langle\mathbb{N}$, prev $\rangle$ be two Soset $\downarrow$-morphisms be defined as $f=i d_{\mathbb{N}}$ and $g=$ succ, where succ is a successor function. Their coequalizer in Rset $\downarrow$ is $e:\langle\mathbb{N}$, prev $\rangle \rightarrow\left\langle C, R_{C}\right\rangle$, where $C=\{*\}$ and $R_{C}=\{\langle *, *\rangle\}$, i.e. a singleton ordered by identity. However, $\left\langle C, R_{C}\right\rangle$ fails to be a Soset $\downarrow$-object, because $R_{C}$ is reflexive. Moreover, no other function may in the same time coequalize functions $f$ and $g$ and stay monotone. Therefore, they have no coequalizer in Soset $\downarrow$. 
Proof of Theorem 25: The coequalizer of two morphisms $f, g: A_{R} \rightarrow B_{R}$ in Soset $_{\mathbf{w f}} \downarrow$ is $e: B_{R} \rightarrow C_{R}$, where $e$ is the coequalizer of $f$ and $g$ in Set and a relation $R_{C}=e\left(R_{B}\right)$. If all descending chains in $R_{B}$ are finite, for any $b_{1}, b_{2} \in B$, if $b_{1}<b_{2}$ then $b_{1} \not \equiv b_{2}$, where $\equiv$ is the equivalence relation as in Lemma 55. To prove this fact, let us assume that there are $b_{1}, b_{2} \in B$ such that $b_{1}<b_{2}$, the proof goes by induction on the length of the descending chain lower to $b_{1}$ wrt. $<$. In the base case let for all $b \in B, b \nless b_{1}$. By contradiction let $b_{1} \equiv b_{2}$. Due to Lemma 55 , since $b_{1} \equiv b_{2}$ and $b_{1}<b_{2}$, there must exist $b_{1}^{\prime}<b_{1}$. Contradiction. In the induction step let us assume that for all $b<b_{1}$, $b \not \equiv b_{1}$. Again by contradiction, let $b_{1} \equiv b_{2}$. Using once more Lemma 55 , since $b_{1} \equiv b_{2}$ and $b_{1}<b_{2}$, we get the existence of $b_{1}^{\prime}<b_{1}$, that $b_{1}^{\prime} \equiv b_{1}$. Contradiction. Therefore, irreflexivity of well-founded $R_{B}$ guarantees irreflexivity of $e\left(R_{B}\right)$ in Soset $_{\mathrm{wf}} \downarrow$.

Proof of Theorem 26: The empty set ordered by the empty relation is an initial object in all above-listed categories. A binary coproduct of $\left\langle A, R_{A}\right\rangle$ and $\left\langle B, R_{B}\right\rangle$ is $\left\langle A \uplus B, R_{A} \uplus R_{B}\right\rangle$. Other finite and infinite coproducts are defined in the same way.

Proof of Theorem 40: The category Soset ${ }_{\mathrm{wf}} \downarrow$ is embeddable into AlgSigDep as a full subcategory (of sort-symbols-only signatures); thus, by Theorem 14, the final object does not exist.

Proof of Theorem 41: Given two AlgSigDep-morphisms $f, g: \Sigma_{<}^{A} \rightarrow \Sigma_{<}^{B}$, let $e: C \rightarrow \operatorname{Symb}\left(\Sigma_{<}^{A}\right)$ be the equalizer of

$$
\operatorname{Symb}(f), \operatorname{Symb}(g): \operatorname{Symb}\left(\Sigma_{<}^{A}\right) \rightarrow \operatorname{Symb}\left(\Sigma_{<}^{B}\right)
$$

in Set. The equalizer of $f$ and $g$ in AlgSigDep is

$$
\boldsymbol{\operatorname { R e c }}_{\Sigma_{<}^{A}}(e): \boldsymbol{\operatorname { R e c }}(e) \rightarrow \Sigma_{<}^{A}
$$

See Def. 37 for a definition of signature morphism reconstruction. See also a proof of Theorem 16 showing that the equalizer from Set is also the equalizer in Soset $_{\mathrm{wf}} \downarrow$.

Proof of Theorem 43: A coequalizer of two morphisms $f, g:\left\langle\Sigma_{A},<_{A}\right\rangle \rightarrow$ $\left\langle\Sigma_{B},<_{B}\right\rangle$ in AlgSigDep is $e:\left\langle\Sigma_{B},<_{B}\right\rangle \rightarrow\left\langle\Sigma_{C},<_{C}\right\rangle$, where $e$ is the coequalizer of $f$ and $g$ in AlgSig and the strict order $<_{C}=\operatorname{Symb}(e)\left(<_{B}\right)$ (cf. Theorem 16). The initial object in AlgSigDep is the empty signature with the empty relation. Binary coproducts in AlgSigDep are binary coproducts in AlgSig ordered by the union of the component orders. Other finite and infinite coproducts are defined in the same way. 